\title{
ANNIHILATOR, COMPLETENESS AND CONVERGENCE OF WAVELET SYSTEM
}

\author{
KWOK-PUN HO
}

\begin{abstract}
We show that if $\{\varphi\}_{Q \in \mathcal{Q}} \in \bigcap \mathcal{M}_{\alpha}\left(\mathbb{R}^{n}\right)$ is a frame and $\left\{\psi_{Q}\right\}_{Q \in \mathcal{Q}} \in$ $\bigcap \mathcal{M}_{\alpha}\left(\mathbb{R}^{n}\right)$ is its dual frame (for the definition of $\mathcal{M}_{\alpha}\left(\mathbb{R}^{n}\right)$, see Definition 2.1), where $\mathcal{Q}$ is the collection of dyadic cubes, then for any $f \in \mathcal{S}^{\prime}\left(\mathbb{R}^{n}\right)$, there exists a sequence of polynomials, $P_{L, L^{\prime}, L^{\prime \prime}}$, such that

$$
\lim _{L, L^{\prime}, L^{\prime \prime} \rightarrow \infty}\left\{\sum_{-L^{\prime} \leq i \leq L} \sum_{|k| \leq \delta(i) 2^{L^{\prime \prime}}}\left\langle f, \psi_{Q_{i, k}}\right\rangle \varphi_{Q_{i, k}}-P_{L, L^{\prime}, L^{\prime \prime}}\right\}=f
$$

in the topology of $\mathcal{S}^{\prime}\left(\mathbb{R}^{n}\right)$, where $\delta(i)=\max \left(2^{i}, 1\right)$. We prove this result by explicitly constructing the polynomials $P_{L, L^{\prime}, L^{\prime \prime}}$. Furthermore, using the above result, we assert that the linear span of the one-dimensional wavelet system is dense in a function space if and only if the dual space of this function space has an trivial intersection with the set of polynomials. This is proved by using the annihilator of the one-dimensional wavelet system.
\end{abstract}

\section{$\S 1$. Introduction}

The purpose of this paper is to prove Theorems 2.1, 3.1, 3.2 and 3.3, as presented in Section 2 and Section 3 and some of theirs generalizations. We show convergence results for frame expansion and wavelet expansion of distributions in Theorems 2.1 and 3.1, respectively. We present one of our main results, Theorem 2.1, in term of frame expansion and consider the corresponding result for wavelet as a special case in Theorem 3.1. Although the result for wavelet is a special case of the result for frame, the convergence of wavelet expansion of Schwartz distribution provides something new on the study of one-dimensional wavelets. Using this theorem, we can determine the annihilator of the wavelet system (see (1.14) for the definition) and establish a condition that guarantees the completeness of wavelet system on any function space in Theorems 3.2 and 3.3.

Received April 25, 2006.

Revised October 16, 2006.

2000 Mathematics Subject Classification: Primary 42B35, 42C15, 42C40; Secondary 46E35, 47B38. 
We begin with some notation and preparations for presenting our main results. Let $L^{p}\left(\mathbb{R}^{n}\right), 1 \leq p \leq \infty$, denote the Lebesgue spaces on $\mathbb{R}^{n}$ and $\mathcal{P}\left(\mathbb{R}^{n}\right)$ be the space of polynomials on $\mathbb{R}^{n}$. We denote the space of Schwartz functions by $\mathcal{S}\left(\mathbb{R}^{n}\right)$. Let $\mathcal{S}_{0}\left(\mathbb{R}^{n}\right)$ consist of Schwartz functions, $g(x)$, satisfying

$$
\int_{\mathbb{R}^{n}} x^{\lambda} g(x) d x=0 \quad \text { for all } \lambda=\left(\lambda_{1}, \ldots, \lambda_{n}\right) \in \mathbb{N}^{n} .
$$

Let $\mathcal{P}_{d}(\mathbb{R})$ be the set of polynomials of degree at most $d, d \in \mathbb{N}$, and $\mathcal{P}(\mathbb{R})=\mathcal{P}_{\infty}(\mathbb{R})=\bigcup_{d \in \mathbb{N}} \mathcal{P}_{d}(\mathbb{R})$ be the set of polynomials.

The dual spaces of $\mathcal{S}\left(\mathbb{R}^{n}\right)$ and $\mathcal{S}_{0}\left(\mathbb{R}^{n}\right)$ are $\mathcal{S}^{\prime}\left(\mathbb{R}^{n}\right)$ (Schwartz Distribution) and $\mathcal{S}_{0}^{\prime}\left(\mathbb{R}^{n}\right)$, respectively. For any $f \in \mathcal{S}^{\prime}\left(\mathbb{R}^{n}\right), \hat{f}$ is the Fourier transform of $f$ and we call the support of $\hat{f}$ the Fourier support of $f$. Let $\langle\cdot, \cdot\rangle$ be the pairing between $\mathcal{S}^{\prime}\left(\mathbb{R}^{n}\right)$ and $\mathcal{S}\left(\mathbb{R}^{n}\right)$. In order to have a pairing that is compatible with the inner product on $L^{2}\left(\mathbb{R}^{n}\right)$, we endow $\mathcal{S}^{\prime}\left(\mathbb{R}^{n}\right)$ with the antilinear structure; that is, for any $f \in \mathcal{S}^{\prime}\left(\mathbb{R}^{n}\right), a, b \in \mathbb{C}$, and $\psi, \phi \in \mathcal{S}\left(\mathbb{R}^{n}\right)$, we have $\langle f, a \phi+b \psi\rangle=\bar{a}\langle f, \phi\rangle+\bar{b}\langle f, \psi\rangle$ where $\bar{a}$ is the complex conjugate of $a$. For instance, the action of the Schwartz distribution, $f(x)=x^{\lambda}, \lambda \in \mathbb{N}^{n}$, on an arbitrary $\psi \in \mathcal{S}\left(\mathbb{R}^{n}\right)$ is given by $\left\langle x^{\lambda}, \psi\right\rangle=\int_{\mathbb{R}} x^{\lambda} \overline{\psi(x)} d x$.

If $f \in L^{p}\left(\mathbb{R}^{n}\right)$ and $g \in L^{p^{\prime}}\left(\mathbb{R}^{n}\right)$ with $\frac{1}{p}+\frac{1}{p^{\prime}}=1,1 \leq p, p^{\prime} \leq \infty$, then $\langle\cdot, \cdot\rangle$ reduces to

$$
\langle f, g\rangle=\int_{\mathbb{R}^{n}} f(x) \overline{g(x)} d x .
$$

We recall the definition of frame from [8], Section 8.1. For a given index set, $\Gamma$, a family of functions, $\left\{\varphi_{\gamma}\right\}_{\gamma \in \Gamma} \in L^{2}\left(\mathbb{R}^{n}\right)$, is a frame on $L^{2}\left(\mathbb{R}^{n}\right)$, if there exist two constants, $C_{2} \geq C_{1}>0$, such that, for any $f \in L^{2}\left(\mathbb{R}^{n}\right)$,

$$
C_{1}\|f\|_{L^{2}}^{2} \leq \sum_{\gamma \in \Gamma}\left|\left\langle f, \varphi_{\gamma}\right\rangle\right|^{2} \leq C_{2}\|f\|_{L^{2}}^{2} .
$$

It is obvious that a frame is complete in $L^{2}\left(\mathbb{R}^{n}\right)$. That is, the linear span of $\left\{\varphi_{\gamma}\right\}_{\gamma \in \Gamma}$ is dense in $L^{2}\left(\mathbb{R}^{n}\right)$.

If $\left\{\varphi_{\gamma}\right\}_{\gamma \in \Gamma}$ is a frame on $L^{2}\left(\mathbb{R}^{n}\right)$, then the associated operator, $\mathrm{S}$ : $L^{2}\left(\mathbb{R}^{n}\right) \rightarrow L^{2}\left(\mathbb{R}^{n}\right)$,

$$
\mathrm{S}(f)=\sum_{\gamma \in \Gamma}\left\langle f, \varphi_{\gamma}\right\rangle \varphi_{\gamma}, \quad f \in L^{2}\left(\mathbb{R}^{n}\right),
$$

is invertible on $L^{2}\left(\mathbb{R}^{n}\right)$. 
Define $\psi_{\gamma}$, by $\psi_{\gamma}=\mathrm{S}^{-1}\left(\varphi_{\gamma}\right)$. The family of functions, $\left\{\psi_{\gamma}\right\}_{\gamma \in \Gamma}$, is called

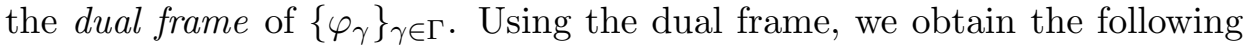
frame expansion for $f \in L^{2}\left(\mathbb{R}^{n}\right)$,

$$
\begin{aligned}
f & =\mathrm{S}\left(\mathrm{S}^{-1}(f)\right)=\sum_{\gamma \in \Gamma}\left\langle f, \varphi_{\gamma}\right\rangle \mathrm{S}^{-1}\left(\varphi_{\gamma}\right)=\sum_{\gamma \in \Gamma}\left\langle f, \varphi_{\gamma}\right\rangle \psi_{\gamma} \\
& =\mathrm{S}^{-1}(\mathrm{~S}(f))=\sum_{\gamma \in \Gamma}\left\langle f, \mathrm{~S}^{-1}\left(\varphi_{\gamma}\right)\right\rangle \varphi_{\gamma}=\sum_{\gamma \in \Gamma}\left\langle f, \psi_{\gamma}\right\rangle \varphi_{\gamma} .
\end{aligned}
$$

Let $\mathcal{Q}$ denote the set of dyadic cubes on $\mathbb{R}^{n}$,

$$
\mathcal{Q}=\left\{Q_{i, k}: i \in \mathbb{Z}, k \in \mathbb{Z}^{n}\right\} \quad \text { with } Q_{i, k}=2^{-i}\left([0,1]^{n}+k\right) .
$$

For each $Q=Q_{i, k}$, we denote the length of $Q, 2^{-i}$, by $l(Q)$, the Lebesgue measure of $Q, 2^{-i n}$, by $|Q|$ and its "left-corner", $2^{-i} k$, by $x_{Q}$.

The function $\varphi(x)$ is called a wavelet for $L^{2}(\mathbb{R})$ if

$$
\varphi_{i, k}(x)=2^{i / 2} \varphi\left(2^{i} x-k\right), \quad i \in \mathbb{Z}, k \in \mathbb{Z},
$$

is an orthonormal basis of $L^{2}(\mathbb{R})$. We call $2^{i}$ and $2^{-i} k$ the dilation and the translation of $\varphi_{i, k}$, respectively.

Moreover, $\left\{\varphi_{Q}\right\}_{Q}$ is a wavelet-type frame if $\varphi_{Q}(x)=\varphi_{i, k}(x)$ when $Q=$ $Q_{i, k}$, where $\varphi_{i, k}(x)=2^{i n / 2} \varphi\left(2^{i} x-k\right)$. Roughly speaking, a wavelet-type frame is a wavelet without orthogonality.

In one of our main results, Theorem 2.1, we establish the convergence of frame expansion of Schwartz distribution when the frame satisfies $\left\{\varphi_{Q}\right\}_{Q \in \mathcal{Q}},\left\{\psi_{Q}\right\}_{Q \in \mathcal{Q}} \in \bigcap_{\alpha>0} \mathcal{M}_{\alpha}\left(\mathbb{R}^{n}\right)$ (for the definition of $\mathcal{M}_{\alpha}\left(\mathbb{R}^{n}\right)$, see Definition 2.1). The precise result is given in Section 2.

As wavelet provides an important example on the convergence of frame expansion of distributions and some results from wavelet give us an important motivation, in this introduction, we are going to have a short overview on the convergence of one-dimensional wavelet expansion and some of its related topics.

There are many references on the convergence of wavelet expansions. One of the pioneer results was obtained by Meyer in [15]. He proved that the wavelet expansion of $f \in L^{p}(\mathbb{R}), 1<p<\infty$, converges in the sense of $L^{p}(\mathbb{R})$ if the wavelet is $r$-regular (see [15] for the definition of $r$-regular). Then, we turned to the pointwise convergence of wavelet expansion. Walter [22] showed that the wavelet expansion of a function $f \in L^{1}(\mathbb{R}) \cap L^{2}(\mathbb{R})$ 
converges to $f$ pointwisely at every point of continuity of $f$ and uniformly on those intervals where $f$ is continuous if the wavelet is $r$-regular. Later, Kelly, Kon and Raphael [12], [13] extended the result in [22] by relaxing the conditions imposed on the wavelet and proved the pointwise convergence of wavelet expansion of $f \in L^{p}(\mathbb{R})$ with $1<p<\infty$. In [26], Zayed further generalized the pointwise convergence of wavelet expansion for wavelets that are not necessarily integrable on $\mathbb{R}$.

We are interested in the convergence of the truncated wavelet expansion of $f \in \mathcal{S}^{\prime}(\mathbb{R})$. Suppose that $\varphi(x) \in \mathcal{S}(\mathbb{R})$ is a wavelet for $L^{2}(\mathbb{R})$. We investigate whether the truncated wavelet expansion,

$$
\sum_{-L^{\prime} \leq i \leq L} \sum_{|k| \leq \delta(i) 2^{L^{\prime \prime}}}\left\langle f, \varphi_{i, k}\right\rangle \varphi_{i, k}, \quad f \in \mathcal{S}^{\prime}(\mathbb{R}),
$$

converges to $f$ in $\mathcal{S}^{\prime}(\mathbb{R})$ as $L, L^{\prime}, L^{\prime \prime}$ go to infinity $\left(L, L^{\prime}\right.$ and $L^{\prime \prime}$ are taken independently), where $\delta(i)=\max \left(2^{i}, 1\right)$.

As we are studying the convergence of wavelet expansion in $\mathcal{S}^{\prime}(\mathbb{R})$, it is important to declare the topology assigned to $\mathcal{S}^{\prime}(\mathbb{R})$. Throughout this article, except Section 5.4, the space of Schwartz distribution, $\mathcal{S}^{\prime}(\mathbb{R})$, is endowed with the weak-star topology inherited from $\mathcal{S}(\mathbb{R})$ (see [2], Chapter V, Section 1, Definition 1.1). That is, the open base of $\mathcal{S}^{\prime}(\mathbb{R})$ consists of $\left\{f \in \mathcal{S}^{\prime}(\mathbb{R}):\left|\left\langle f, \psi_{1}\right\rangle\right|<\epsilon_{1}, \ldots,\left|\left\langle f, \psi_{m}\right\rangle\right|<\epsilon_{m}\right\}$, for some $\psi_{1}, \ldots, \psi_{m} \in \mathcal{S}(\mathbb{R})$ and $\epsilon_{1}, \ldots, \epsilon_{m}>0$. It is easy to see that, with the weak-star topology, $\mathcal{S}^{\prime}(\mathbb{R})$ is a locally convex topological vector spaces.

The weak-star topology is the usual topology assigned to $\mathcal{S}^{\prime}(\mathbb{R})$. For example, the dual space of $\mathcal{S}^{\prime}(\mathbb{R})$ is $\mathcal{S}(\mathbb{R})$ (see [2], Chapter V, Section 1, Theorem 1.3) and the Fourier transform is a continuous linear mapping in $\mathcal{S}^{\prime}(\mathbb{R})$ if $\mathcal{S}^{\prime}(\mathbb{R})$ is endowed with the weak-star topology.

Of course, we can endow $\mathcal{S}^{\prime}(\mathbb{R})$ with a topology different from the weakstar topology. In Section 5.4, we briefly mention the convergence of the frame expansion for Schwartz distribution under the "inductive limit topology" (for the definition of inductive limit topology, see [2], Chapter IV, Section 5).

Before we proceed to the discussion on introducing the floating polynomials in our wavelet expansion, we would like to say a few words on the truncation set,

$$
T\left(L, L^{\prime}, L^{\prime \prime}\right)=\left\{(i, k): i \in \mathbb{Z},-L^{\prime} \leq i \leq L ; k \in \mathbb{Z},|k| \leq \delta(i) 2^{L^{\prime \prime}}\right\} .
$$


In this article, the truncation set is $T\left(L, L^{\prime}, L^{\prime \prime}\right)$ although it seems that it is more reasonable to consider

$$
\tilde{T}\left(L, L^{\prime}, L^{\prime \prime}\right)=\left\{(i, k): i \in \mathbb{Z},-L^{\prime} \leq i \leq L ; k \in \mathbb{Z},\left|2^{-i} k\right| \leq 2^{L^{\prime \prime}}\right\}
$$

as the truncation set because $2^{-i} k$ corresponds to the translation of $\varphi_{i, k}$.

The use of $T\left(L, L^{\prime}, L^{\prime \prime}\right)$ is supported by Lemma 7.1 in Section 7. Roughly speaking, in order to have the fastest rate of convergence, the truncation set should collect those "relatively large" wavelet coefficients, $\left\langle f, \varphi_{i, k}\right\rangle, f \in$ $\mathcal{S}(\mathbb{R})$. By Lemma 7.1, the decay for $\left\langle f, \varphi_{i, k}\right\rangle$ is different according to $i \geq$ 0 and $i<0$. We, therefore, introduce $\delta(i)$ to balance the disproportion between $i \geq 0$ and $i<0$. The formulation of $\delta(i)$ and the detail of using $\delta(i)$ in our analysis can be found in Section 7.

In Section 8.1, we will discuss the convergence of frame expansion with $\tilde{T}\left(L, L^{\prime}, L^{\prime \prime}\right)$ as the truncation set. In fact, our results are still valid if we take $\tilde{T}\left(L, L^{\prime}, L^{\prime \prime}\right)$ as the truncation set. The shortcoming of using $\tilde{T}\left(L, L^{\prime}, L^{\prime \prime}\right)$ is that we have a slower rate of convergence (see (7.11) and (8.1)).

Now, we analyze why we need to modify our wavelet expansion of $f \in$ $\mathcal{S}^{\prime}(\mathbb{R})$ by adding a sequence of "floating polynomials". Let us examine the expansion, (1.5), and see what problem arises if we solely use (1.5) for $f \in \mathcal{S}^{\prime}(\mathbb{R})$. Suppose that for any $f \in \mathcal{S}^{\prime}(\mathbb{R})$, we have

$$
\lim _{L, L^{\prime}, L^{\prime \prime} \rightarrow \infty} \sum_{-L^{\prime} \leq i \leq L} \sum_{|k| \leq \delta(i) 2^{L^{\prime \prime}}}\left\langle f, \varphi_{i, k}\right\rangle \varphi_{i, k}=f
$$

in $\mathcal{S}^{\prime}(\mathbb{R})$.

First, the proof of Theorem 3.4 in Section 2.3 of Hernández and Weiss [8] shows that if $\varphi$ is a wavelet for $L^{2}(\mathbb{R})$ and satisfies

$$
|\varphi(x)| \leq \frac{1}{(1+|x|)^{1+s}}, \quad \text { for some } s>[s] \geq 0,
$$

where $[s]$ is the integral part of $s$ and $\varphi^{(m)} \in L^{\infty}(\mathbb{R})$ for $m=1,2, \ldots,[s]$, then all moments up to order $[s]$ are zero; that is,

$$
\int_{\mathbb{R}} x^{\lambda} \varphi(x) d x=0 \quad \text { for } \lambda \in \mathbb{N} \text { and } 0 \leq \lambda \leq[s] .
$$

As $\varphi \in \mathcal{S}(\mathbb{R}), \varphi$ satisfies (1.8) with $s=\infty$. If we apply (1.6) to the Schwartz distribution, $f(x)=x^{\lambda}, 0 \leq \lambda<\infty$, then the left-hand side of 
(1.6) is equal to zero for any $L, L^{\prime}$ and $L^{\prime \prime}$ while the right-hand side is not. Thus, the truncated wavelet expansion, (1.5), cannot converge to $f$ in $\mathcal{S}^{\prime}(\mathbb{R})$.

The Littlewood-Paley analysis on $\mathbb{R}^{n}$ (see [16], Chapter 1 , Section 3) gives us an inspiration on introducing the floating polynomials. Specifically, the Littlewood-Paley analysis involves a function, $\psi \in \mathcal{S}_{0}\left(\mathbb{R}^{n}\right)$, satisfying

$$
\begin{gathered}
\operatorname{supp} \hat{\psi}(\xi) \subset\left\{\xi \in \mathbb{R}^{n}: 1 / 2 \leq|\xi| \leq 2\right\} \\
\sum_{j=-\infty}^{\infty} \hat{\psi}\left(2^{j} \xi\right)=1 \quad \text { if } \xi \neq 0 .
\end{gathered}
$$

For any $f \in \mathcal{S}^{\prime}\left(\mathbb{R}^{n}\right)$, we define $\triangle_{j}(f)=f * \psi_{j}$ where $\psi_{j}(x)=2^{n j} \psi\left(2^{j} x\right)$ and $f * \psi_{j}$ is the convolution of $f$ and $\psi_{j}$.

The following well-known theorem is the fundamental result on the convergence of the Littlewood-Paley analysis for $f \in \mathcal{S}^{\prime}\left(\mathbb{R}^{n}\right)$.

Proposition 1.1. If $f$ is a Schwartz distribution, there exist an integer, $N$, and a sequence, $P_{q}(x)$, of polynomials of degrees less than or equal to $N$ such that

$$
f=\lim _{q \rightarrow+\infty}\left\{\sum_{j=-q}^{0} \triangle_{j}(f)-P_{q}\right\}+\sum_{j=1}^{\infty} \triangle_{j}(f) .
$$

The above result was observed by Peetre in [18], see pp. 51-54 of [18]. For the proof of the above proposition, the reader is referred to [16] Chapter 1, Section 3, Proposition 1.5. Moreover, in Chapter 2 of [16], there are some ideas on the convergence of the wavelet expansion of $f(x)=|x|^{s}$ when $0<s<1$ or $m<s<m+1, m \in \mathbb{N}$.

There is another similar result in [5] and [6], where Frazier and Jawerth showed that if $\varphi, \psi \in \mathcal{S}\left(\mathbb{R}^{n}\right)$ satisfy

$$
\begin{gathered}
\operatorname{supp} \hat{\psi}(\xi), \operatorname{supp} \hat{\varphi}(\xi) \subset\left\{\xi \in \mathbb{R}^{n}: 1 / 2 \leq|\xi| \leq 2\right\} \\
\sum_{j=-\infty}^{\infty} \overline{\hat{\varphi}\left(2^{j} \xi\right)} \hat{\psi}\left(2^{j} \xi\right)=1 \quad \text { if } \xi \neq 0,
\end{gathered}
$$

then, for any $f \in \mathcal{S}^{\prime}\left(\mathbb{R}^{n}\right) / \mathcal{P}\left(\mathbb{R}^{n}\right)$ (the space of Schwartz distributions modulo polynomials with the weak-star topology induced by $\left.\mathcal{S}_{0}\left(\mathbb{R}^{n}\right)\right)$,

$$
\sum_{i \in \mathbb{Z}, k \in \mathbb{Z}^{n}}\left\langle f, \varphi_{i, k}\right\rangle \psi_{i, k}
$$


converges to $f$ in $\mathcal{S}^{\prime}\left(\mathbb{R}^{n}\right) / \mathcal{P}\left(\mathbb{R}^{n}\right)$, where $\varphi_{i, k}(x)=2^{i n / 2} \varphi\left(2^{i} x-k\right)$ and $\psi_{i, k}(x)=2^{i n / 2} \psi\left(2^{i} x-k\right)$. Frazier and Jawerth used the Littlewood-Paley analysis and Shannon's sampling theorem to prove this result (see Lemma 2.1 of [5]). Therefore, the compactness of the Fourier supports of $\varphi$ and $\psi$ is essential in proving the convergence of (1.13). Notice that we do not make any assumption on the Fourier support of the wavelet function, $\varphi(x)$, in this paper. Therefore, Shannon's sampling theorem and the LittlewoodPaley analysis can no longer be used as they were by Frazier and Jawerth [5], [6] and Meyer [16].

As mentioned in [16], we need a renormalization to fix the "infrared divergence". The correct renormalization is a sequence of floating polynomials (the polynomials, $P_{q}$, in Proposition 1.1). In this paper, we prove the convergence of wavelet expansion for $f \in \mathcal{S}^{\prime}(\mathbb{R})$ and construct the corresponding floating polynomials. The precise meaning and the construction of these floating polynomials for wavelet expansion of Schwartz distributions are given in Theorem 3.1.

The convergence of wavelet expansion of Schwartz distribution provides some new insight for one-dimensional wavelet system. By using the above result on the convergence of wavelet expansion, we can determine the annihilator of the wavelet system. That is, for any function space $B$ satisfying $\mathcal{S}(\mathbb{R}) \hookrightarrow B \hookrightarrow \mathcal{S}^{\prime}(\mathbb{R})$, we search for an explicit expression of

$$
\left\{f \in B^{*}:\left\langle f, \varphi_{i, k}\right\rangle=0, \forall i, k \in \mathbb{Z}\right\},
$$

where $B^{*}$ denotes the dual space of $B$.

The interest in the completeness of the wavelet system, $\left\{\varphi_{i, k}\right\}_{i, k \in \mathbb{Z}}$, on function spaces is the reason for the study of the annihilator. Recall that if the wavelet system is complete in a function space, $B$, then the linear span of the wavelet system is dense in $B$. By using the Hahn-Banach Theorem, the annihilator provides a characterization on whether any function space, $B$, has the wavelet system as a complete system.

If we take $B=L^{2}(\mathbb{R})$, it is obvious that the annihilator is equal to the trivial set. That is, $\{0\}$. Nevertheless, if we consider $B=\mathcal{S}(\mathbb{R}),(1.8)$ shows that

$$
\mathcal{P}_{[s]}(\mathbb{R}) \subseteq\left\{f \in \mathcal{S}^{\prime}(\mathbb{R}):\left\langle f, \varphi_{i, k}\right\rangle=0, \forall i, k \in \mathbb{Z}\right\},
$$

if $\varphi$ satisfies (1.7).

In this paper, we show that, by using the convergence of wavelet expansion for distributions, we have an identity in (1.15). Furthermore, once 
we determine that the annihilator for the wavelet system is equal to the set of polynomials, we can use the Hahn-Banach theorem to deduce a condition that guarantees the completeness of the wavelet system, $\left\{\varphi_{i, k}\right\}_{i, k \in \mathbb{Z}}$, on function spaces. We prove that a locally convex function space, $B$, satisfying $\mathcal{S}(\mathbb{R}) \hookrightarrow B \hookrightarrow \mathcal{S}^{\prime}(\mathbb{R})$, has $\left\{\varphi_{i, k}\right\}_{i, k \in \mathbb{Z}}$ as a complete system if and only if

$$
B^{*} \cap \mathcal{P}(\mathbb{R})=\{0\} .
$$

Other than the study of completeness of the wavelet system, the annihilator is also related to the Fix-Strang condition. Before we go into details of it, let us recall some important notions in wavelet theory.

Let $\Phi$ be a scaling function and $\varphi$ be its wavelet. The corresponding multiresolution analysis, $\left\{V_{j}\right\}_{j \in \mathbb{Z}} \subset L^{2}(\mathbb{R})$, is given by

$$
V_{j}=\left\{\sum_{k \in \mathbb{Z}} \alpha_{k} \Phi\left(2^{j} x-k\right): \sum_{k \in \mathbb{Z}}\left|\alpha_{k}\right|^{2}<\infty\right\} .
$$

From Theorem 1.6 in Section 2.1 of [8], we can compute the tail of the $M R A, \bigcap_{j \in \mathbb{Z}} V_{j}$. We have $\bigcap_{j \in \mathbb{Z}} V_{j}=\{0\}$. That the tail of the MRA equals to the trivial set is an important property because with this identity, we can decompose $L^{2}(\mathbb{R})$ as $L^{2}(\mathbb{R})=\bigoplus_{j=-\infty}^{\infty} W_{j}$, where $W_{j}$ is the closure of the linear span of $\{\varphi(\cdot-k)\}_{k \in \mathbb{Z}}$. Precisely, the family $\{\varphi(\cdot-k)\}_{k \in \mathbb{Z}}$ is an orthogonal basis of $W_{j}$. Furthermore, $W_{j}$ is the orthogonal complement of $V_{j+1}$ from $V_{j}$. That is $V_{j+1}=V_{j} \oplus W_{j}$.

In order to see whether we can use MRA to study function spaces other than $L^{2}(\mathbb{R})$, for example, $\mathcal{S}^{\prime}(\mathbb{R})$, we alter the definition of $V_{j},(1.16)$, by

$$
T_{j}=\left\{f: f(x)=\sum_{k \in \mathbb{Z}} a_{k} \Phi\left(2^{j} x-k\right) ;\left|a_{k}\right|=O\left(|k|^{l}\right) \text { for some } l \in \mathbb{Z}\right\}
$$

The above definition of $T_{j}$ is provided by Walter in [23].

Unfortunately, the tail of $\left\{T_{j}\right\}_{j \in \mathbb{Z}}, \bigcap_{j \in \mathbb{Z}} T_{j}$, is not a trivial set. In fact, we find that

$$
\mathcal{P}_{p}(\mathbb{R}) \subseteq \bigcap_{j \in \mathbb{Z}} T_{j}
$$

where $p \in \mathbb{N} \cup\{\infty\}$ depends on the order of vanishing moment the wavelet satisfied. We have the following result which is a part of Theorem 7.4 of $[14]$. 
Theorem 1.2. Let $p \in \mathbb{N} \cup\{\infty\}, \varphi$ and $\Phi$ be a wavelet and a scaling function that generate an orthogonal basis. Suppose that $|\varphi(x)|=O((1+$ $\left.\left.x^{2}\right)^{-p / 2-1}\right)$ and $|\Phi(x)|=O\left(\left(1+x^{2}\right)^{-p / 2-1}\right)$. The following statement are equivalent:

(1) The wavelet $\varphi$ satisfies condition (1.8) with $p=s$.

(2) For any $0 \leq l \leq p, l \in \mathbb{N}$,

$$
q_{l}(t)=\sum_{k=-\infty}^{\infty} k^{l} \Phi(t-k)
$$

is a polynomial of degree $l$.

The proof of the above theorem relies heavily on Fourier transform. The hypothesis, (2), in Theorem 1.2 is called the Fix-Strang condition (see [4]). With the definition of $T_{j}$, the inclusion, (1.18), is another expression of the Fix-Strang condition.

In this paper, we prove that if $\Phi, \varphi \in \mathcal{S}(\mathbb{R})$, then the tail of $\left\{T_{j}\right\}_{j \in \mathbb{Z}}$ is equal to the set of polynomials. That is,

$$
\mathcal{P}(\mathbb{R})=\bigcap_{j \in \mathbb{Z}} T_{j}
$$

Moreover, we obtain another representation of the set on the right-hand side of (1.20) where this representation does not involve the scaling function. Specifically, when the wavelet, $\varphi \in \mathcal{S}(\mathbb{R})$, we show that the sets in (1.20) also equal to the annihilator for the wavelet system. Furthermore, it provides a new proof of the Fix-Strang condition where Fourier transform is not involved.

In addition, by using our idea for proving Theorem 2.1, we show another interesting result about "reproducing formula" for Schwartz distributions. A reproducing formula for $\mathcal{S}^{\prime}\left(\mathbb{R}^{n}\right) / \mathcal{P}\left(\mathbb{R}^{n}\right)$ involves two families of functions $\left\{\varphi_{I}\right\}_{I \in \mathcal{I}},\left\{\psi_{I}\right\}_{I \in \mathcal{I}} \subset \mathcal{S}_{0}\left(\mathbb{R}^{n}\right)$ such that

$$
f=\sum_{I \in \mathcal{I}}\left\langle f, \varphi_{I}\right\rangle \psi_{I}, \quad \forall f \in \mathcal{S}^{\prime}\left(\mathbb{R}^{n}\right) / \mathcal{P}\left(\mathbb{R}^{n}\right) .
$$

We call $\left\{\varphi_{I}\right\}_{I \in \mathcal{I}}$ the analyzing family and $\left\{\psi_{I}\right\}_{I \in \mathcal{I}}$ the reconstructing family of (1.21). We prove in Theorem 6.2 that an analyzing family is also a 
reconstructing family, and vice verse, if $\left\{\varphi_{I}\right\}_{I \in \mathcal{I}}$ and $\left\{\psi_{I}\right\}_{I \in \mathcal{I}}$ are "welllocalized". More specifically, if $\left\{\varphi_{I}\right\}_{I \in \mathcal{I}},\left\{\psi_{I}\right\}_{I \in \mathcal{I}} \in \bigcap_{\alpha>0} \mathcal{M}_{\alpha}\left(\mathbb{R}^{n}\right)$ (for the definition of $\mathcal{M}_{\alpha}\left(\mathbb{R}^{n}\right)$, see Definition 2.1) satisfy (1.21), then, we assert that

$$
f=\sum_{I \in \mathcal{I}}\left\langle f, \psi_{I}\right\rangle \varphi_{I}, \quad \forall f \in \mathcal{S}^{\prime}\left(\mathbb{R}^{n}\right) / \mathcal{P}\left(\mathbb{R}^{n}\right) .
$$

We see that the notions of analyzing family and reconstructing family are the same for Schwartz distributions. The precise meaning of the above result is given in Section 6 .

This paper is organized as follows. The main theorem for frame, Theorem 2.1, is presented in Section 2. The main results for one-dimensional wavelets, namely, the convergence, the annihilator and the completeness of one-dimensional wavelets, are given in Section 3. In Section 4, we introduce a class of function spaces, define the order for a distribution in $\mathcal{S}^{\prime}\left(\mathbb{R}^{n}\right)$, and state the convergence theorem for frame expansion of the Schwartz function, $\mathcal{S}\left(\mathbb{R}^{n}\right)$, the proof of Theorem 2.1 is also given in this section. We present some generalizations of Theorems 2.1, 3.1, 3.2 and 3.3 and compute the tail of a generalized MRA (see Section 5.3 for the definition) in Section 5. We state and prove Theorem 6.2 in Section 6. We provide the proof for Theorem 4.1 in Section 7 and consider the convergence of wavelet expansions with truncation sets different from $T\left(L, L^{\prime}, L^{\prime \prime}\right)$ in Section 8.1.

\section{§2. Main results for convergence of frame expansion}

In this Section, we state the main result for frame. In order to control the decay and smoothness of the frame, we introduce the following classes of functions. The following definition is motivated by the definitions of smooth molecules in [6] (pp. 56-57, conditions (3.3)-(3.10)) and vaguelets in [1] (Chapter 8, Section 5, Definition 3).

Definition 2.1. Given a fixed $\epsilon>0$, for $\alpha>0$, we say that $m_{Q}$, $Q \in \mathcal{Q}$, is an homogeneous molecule with order $\alpha$, if it satisfies

$$
\int_{\mathbb{R}^{n}} x^{\lambda} m_{Q}(x) d x=0 \quad \text { if }|\lambda| \leq[\alpha],
$$

where $\lambda=\left(\lambda_{1}, \ldots, \lambda_{n}\right) \in \mathbb{N}^{n}$ and $|\lambda|=\left|\lambda_{1}\right|+\cdots+\left|\lambda_{n}\right|$;

$$
\left|\partial^{\gamma} m_{Q}(x)\right| \leq C_{1}|Q|^{-1 / 2-|\gamma| / n} \frac{1}{\left(1+l(Q)^{-1}\left|x-x_{Q}\right|\right)^{\alpha+n+\epsilon}}
$$


if $\gamma \in \mathbb{N}^{n}$ and $|\gamma| \leq[\alpha]$; and

$$
\begin{aligned}
& \left|\partial^{\gamma} m_{Q}(x)-\partial^{\gamma} m_{Q}(y)\right| \\
& \quad \leq C_{2}|Q|^{-1 / 2-\alpha / n}|x-y|^{\alpha-[\alpha]} \sup _{\left|z-x_{Q}\right| \leq|x-y|} \frac{1}{\left(1+l(Q)^{-1}|x-z|\right)^{\alpha+n+\epsilon}}
\end{aligned}
$$

if $|\gamma|=[\alpha]<\alpha$.

We call $\left\{m_{Q}\right\}_{Q}$ a family of homogeneous molecules. The class of these families of homogeneous molecules is denoted by $\mathcal{M}_{\alpha}\left(\mathbb{R}^{n}\right)$. We define the norm, $\|\cdot\|_{\mathcal{M}_{\alpha}}$, to be $\max \left(\|\cdot\|_{*},\|\cdot\|_{* *}\right)$ where $\left\|\left\{m_{Q}\right\}_{Q}\right\|_{*}$ is the infimum of $C_{1}$ in (2.2) and $\left\|\left\{m_{Q}\right\}_{Q}\right\|_{* *}$ is the infimum of $C_{2}$ in (2.3).

The following result is one of our main theorems. It presents the convergence of frame expansion for Schwartz distribution. Using this result, we determine the annihilator of wavelet system in Section 3.2 and study the completeness of wavelet system in Section 3.3.

The proof of the following theorem is given in Section 4.2.

Theorem 2.1. Let $\left\{\varphi_{Q}\right\}_{Q \in \mathcal{Q}} \in \bigcap_{\alpha>0} \mathcal{M}_{\alpha}\left(\mathbb{R}^{n}\right)$ be a frame on $L^{2}\left(\mathbb{R}^{n}\right)$ and $\left\{\psi_{Q}\right\}_{Q \in \mathcal{Q}} \in \bigcap_{\alpha>0} \mathcal{M}_{\alpha}\left(\mathbb{R}^{n}\right)$ be its dual frame. Then, for any $f \in$ $\mathcal{S}^{\prime}\left(\mathbb{R}^{n}\right)$, there exist an integer, $N$, and a sequence of floating polynomials, $P_{L, L^{\prime}, L^{\prime \prime}}(x)$, of degrees less than or equal to $N$ such that

$$
\lim _{L, L^{\prime}, L^{\prime \prime} \rightarrow \infty}\left\{\sum_{i=-L^{\prime}}^{L} \sum_{|k| \leq \delta(i) 2^{L^{\prime \prime}}}\left\langle f, \psi_{Q_{i, k}}\right\rangle \varphi_{Q_{i, k}}-P_{L, L^{\prime}, L^{\prime \prime}}\right\}=f
$$

in the topology of $\mathcal{S}^{\prime}\left(\mathbb{R}^{n}\right)$ (the weak-star topology inherited from $\mathcal{S}\left(\mathbb{R}^{n}\right)$ ). More precisely, we have

$$
\begin{aligned}
P_{L, L^{\prime}, L^{\prime \prime}}(x) & =P_{L, L^{\prime}, L^{\prime \prime}}^{\theta}(x) \\
& =-\sum_{0 \leq|\lambda| \leq N}\left(\left\langle f, \theta_{\lambda}\right\rangle-\sum_{i=-L^{\prime}}^{L} \sum_{|k| \leq \delta(i) 2^{L^{\prime \prime}}}\left\langle\varphi_{Q_{i, k}}, \theta_{\lambda}\right\rangle\left\langle f, \psi_{Q_{i, k}}\right\rangle\right) x^{\lambda},
\end{aligned}
$$

where $\lambda \in \mathbb{N}^{n}$ and $\theta_{\lambda} \in \mathcal{S}\left(\mathbb{R}^{n}\right)$ satisfies

$$
\int_{\mathbb{R}^{n}} x^{\gamma} \theta_{\lambda}(x) d x=\delta_{\gamma \lambda}=\left\{\begin{array}{ll}
1 & \text { if } \gamma=\lambda \\
0 & \text { if } \gamma \neq \lambda,
\end{array} \text { for } \gamma \in \mathbb{N}^{n} \text { and } 0 \leq|\gamma| \leq N\right.
$$


Remark 2.1. Note that if $\varphi \in \mathcal{S}_{0}\left(\mathbb{R}^{n}\right)$ satisfies the Calderón reproducing formula

$$
\sum_{i \in \mathbb{Z}}\left|\hat{\varphi}\left(2^{i} \xi\right)\right|^{2}=1 \quad \text { if } \xi \neq 0 .
$$

Then, there exists an integer, $b$, such that the function, $\varphi\left(2^{b} x\right)$, is a wavelet-

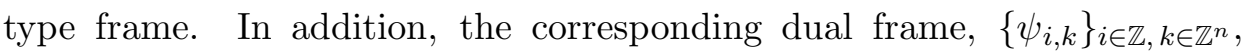
belongs to $\bigcap_{\alpha} \mathcal{M}_{\alpha}\left(\mathbb{R}^{n}\right)$ although $\left\{\psi_{i, k}\right\}_{i \in \mathbb{Z}, k \in \mathbb{Z}^{n}}$ is not necessarily a dilation and translation of a fixed function, see [9]. The results in [9] are for frames with matrix dilations and the above result is obviously a special case. Therefore, it makes sense to assume $\left\{\varphi_{Q}\right\}_{Q \in \mathcal{Q}},\left\{\psi_{Q}\right\}_{Q \in \mathcal{Q}} \in \bigcap_{\alpha} \mathcal{M}_{\alpha}\left(\mathbb{R}^{n}\right)$ in Theorem 2.1.

As $\left\{\varphi_{Q}\right\}_{Q \in \mathcal{Q}}$ is a frame on $L^{2}\left(\mathbb{R}^{n}\right)$ and $\left\{\psi_{Q}\right\}_{Q \in \mathcal{Q}}$ is its dual frame, the functions $\varphi_{Q}$ and $\psi_{Q}$ in (2.4) can be interchanged. We provide a more general result in this direction in Section 6 .

Note that the floating polynomials, $P_{L, L^{\prime}, L^{\prime \prime}} \in \mathcal{S}^{\prime}\left(\mathbb{R}^{n}\right)$, depend continuously and linearly on $f \in \mathcal{S}^{\prime}\left(\mathbb{R}^{n}\right)$.

In Section 8.2, we show that if $\theta_{\lambda}^{1}, \theta_{\lambda}^{2} \in \mathcal{S}\left(\mathbb{R}^{n}\right)$ fulfill condition (2.5), then the corresponding floating polynomials, $P_{L, L^{\prime}, L^{\prime \prime}}^{\theta^{1}}$ and $P_{L, L^{\prime}, L^{\prime \prime}}^{\theta^{2}}$, satisfy $P_{L, L^{\prime}, L^{\prime \prime}}^{\theta^{1}}-P_{L, L^{\prime}, L^{\prime \prime}}^{\theta^{2}} \rightarrow 0$ in $\mathcal{S}^{\prime}\left(\mathbb{R}^{n}\right)$ as $L, L^{\prime}$ and $L^{\prime \prime}$ go to infinity. Therefore, the convergence in (2.4) is independent of the family, $\left\{\theta_{\lambda}\right\}$. Furthermore, in that section, we prove that if $f \in L^{2}\left(\mathbb{R}^{n}\right)$, then the corresponding floating polynomials, $P_{L, L^{\prime}, L^{\prime \prime}}$, converges to zero in $\mathcal{S}^{\prime}\left(\mathbb{R}^{n}\right)$ as $L, L^{\prime}, L^{\prime \prime} \rightarrow \infty$.

Theorem 2.1 is proved by considering the "dual problem". We first shift our attention from the convergence of frame expansion of Schwartz distributions, $\mathcal{S}^{\prime}\left(\mathbb{R}^{n}\right)$, to the convergence of frame expansion of Schwartz functions, $\mathcal{S}\left(\mathbb{R}^{n}\right)$. Then, we try to determine the condition imposed on $g \in \mathcal{S}\left(\mathbb{R}^{n}\right)$ such that

$$
\sum_{i=-L^{\prime}}^{L} \sum_{|k| \leq \delta(i) 2^{L^{\prime \prime}}}\left\langle g, \varphi_{Q_{i, k}}\right\rangle \psi_{Q_{i, k}} \rightarrow g \quad \text { in } \mathcal{S}\left(\mathbb{R}^{n}\right)
$$

as $L, L^{\prime}$ and $L^{\prime \prime}$ go to infinity. We show in Theorem 4.1 that (2.6) holds if and only if $g \in \mathcal{S}\left(\mathbb{R}^{n}\right)$ satisfies the vanishing moment condition, $\int_{\mathbb{R}^{n}} x^{\lambda} g(x) d x=$ 0 for all $\lambda \in \mathbb{N}^{n}$.

It turns out that the above condition is too strong because it requires infinity many vanishing moments. We need a weaker condition which just 
involves finite many moments. To rectify this situation, we consider the convergence of frame expansion under another function spaces, namely, $\mathcal{C}_{\alpha}\left(\mathbb{R}^{n}\right)$ (we definite $\mathcal{C}_{\alpha}\left(\mathbb{R}^{n}\right)$ in Definition 4.1).

Roughly speaking, the frame expansion of $g \in \mathcal{S}\left(\mathbb{R}^{n}\right)$ converges in $\mathcal{C}_{\alpha}\left(\mathbb{R}^{n}\right)$ if $g$ satisfies $\int_{\mathbb{R}^{n}} x^{\lambda} g(x) d x=0$ for $\lambda \in \mathbb{N}^{n}$ and $0 \leq|\lambda| \leq[\alpha]$. The precise statement of the above result is presented in Theorem 4.1.

If $f \in \mathcal{S}^{\prime}\left(\mathbb{R}^{n}\right)$ is of "order" less than or equal to $\alpha$ (the definition of the order of a Schwartz distribution is given in Section 4), then we can apply $f$ on the truncated frame expansion of $g \in \mathcal{S}\left(\mathbb{R}^{n}\right)$. In order to fulfill the vanishing moment condition imposed on $g \in \mathcal{S}\left(\mathbb{R}^{n}\right)$, we need to modify $g$ and this produces the floating polynomials in Theorem 2.1. The space of Schwartz distribution, $\mathcal{S}^{\prime}\left(\mathbb{R}^{n}\right)$, is endowed with the weak-star topology inherited from $\mathcal{S}\left(\mathbb{R}^{n}\right)$. That is, $f_{i} \rightarrow f$ in $\mathcal{S}^{\prime}\left(\mathbb{R}^{n}\right)$ if and only if, for any $\psi \in \mathcal{S}\left(\mathbb{R}^{n}\right),\left\langle f_{i}, \psi\right\rangle \rightarrow\langle f, \psi\rangle$. Therefore, we can obtain our result by duality. Notice that, in [7], pp. 122-125, Frazier et al. also use the duality argument to prove the convergence of the "continuous Calderón reproducing formula" in $\mathcal{S}^{\prime}\left(\mathbb{R}^{n}\right) / \mathcal{P}\left(\mathbb{R}^{n}\right)$.

Our argument for proving Theorem 2.1 can be used to generalize the Littlewood-Paley analysis, the detail is given in [10]. In [10], we can show that Proposition 1.1 still holds when $\psi \in \mathcal{S}\left(\mathbb{R}^{n}\right)$ only satisfies condition (1.10). The reader may wonder whether we can use the generalized Littlewood-Paley analysis to prove the convergence of frame expansion for Schwartz distribution. Although we do not need (1.9) for the generalized Littlewood-Paley analysis, the compactness of the Fourier support of the function, $\varphi$, required by Shannon's formula (see the conditions for identity (3.4)) forbids the use of the generalized Littlewood-Paley analysis in proving Theorem 2.1.

\section{§3. Main results for one-dimensional wavelets}

\subsection{Convergence of wavelet system}

We are particular interested in the consequences of the results in Theorem 2.1 on the one-dimensional wavelets. Since one-dimensional wavelet $\varphi \in \mathcal{S}(\mathbb{R})$ satisfies a very important property

$$
\int_{\mathbb{R}} x^{s} \varphi(x) d x=0, \quad s \in \mathbb{N},
$$

we have the following result for wavelet. 
TheOREM 3.1. Let $\varphi \in \mathcal{S}(\mathbb{R})$ be a wavelet for $L^{2}(\mathbb{R})$. Then, for any $f \in \mathcal{S}^{\prime}(\mathbb{R})$, there exist an integer, $N$, and a sequence of floating polynomials, $P_{L, L^{\prime}, L^{\prime \prime}}(x)$, of degrees less than or equal to $N$ such that

$$
\lim _{L, L^{\prime}, L^{\prime \prime} \rightarrow \infty}\left\{\sum_{-L^{\prime} \leq i \leq L} \sum_{|k| \leq \delta(i) 2^{L^{\prime \prime}}}\left\langle f, \varphi_{i, k}\right\rangle \varphi_{i, k}-P_{L, L^{\prime}, L^{\prime \prime}}\right\}=f
$$

in the topology of $\mathcal{S}^{\prime}(\mathbb{R})$ (the weak-star topology inherited from $\mathcal{S}(\mathbb{R})$ ) where

$$
\begin{aligned}
P_{L, L^{\prime}, L^{\prime \prime}}(x) & =P_{L, L^{\prime}, L^{\prime \prime}}^{\theta}(x) \\
& =-\sum_{0 \leq \lambda \leq N}\left(\left\langle f, \theta_{\lambda}\right\rangle-\sum_{-L^{\prime} \leq i \leq L} \sum_{|k| \leq \delta(i) 2^{L^{\prime \prime}}}\left\langle\varphi_{i, k}, \theta_{\lambda}\right\rangle\left\langle f, \varphi_{i, k}\right\rangle\right) x^{\lambda},
\end{aligned}
$$

$\lambda \in \mathbb{N}$ and $\theta_{\lambda} \in \mathcal{S}(\mathbb{R})$ satisfies

$$
\int_{\mathbb{R}} x^{\gamma} \theta_{\lambda}(x) d x=\delta_{\gamma \lambda}=\left\{\begin{array}{ll}
1 & \text { if } \gamma=\lambda \\
0 & \text { if } \gamma \neq \lambda,
\end{array} \quad \text { for } \gamma \in \mathbb{N} \text { and } 0 \leq \gamma \leq N\right.
$$

The existence of wavelets belonging to $\mathcal{S}(\mathbb{R})$ had been shown by Lemarié and Meyer (see [8], Section 1.4). The Lemarié-Meyer wavelets can be constructed by multiresolution analysis (see [8], Section 2.2, Example D), moreover, the corresponding scaling function also belongs to $\mathcal{S}(\mathbb{R})$.

Although the above result is a special case of Theorem 2.1, it has its own independent interest. This is the first proof on this expected result. The above result for the Lemarié-Meyer wavelets cannot be proved by Shannon's sampling theorem and the Littlewood-Paley analysis, even though the Fourier support of the Lemarie-Meyer wavelets is compact (that is, they are band-limited wavelets). The obstacle is found in the inconsistency between the Fourier support of the Lemarié-Meyer wavelets and the "sampling frequency" given by Shannon's formula. Let $\varphi$ be a Lemarié-Meyer wavelet on $\mathbb{R}$, after expanding $f \in \mathcal{S}^{\prime}(\mathbb{R})$ by the Littlewood-Paley analysis (Proposition 1.1 with $\left.\hat{\psi}=|\hat{\varphi}|^{2}\right)$; that is,

$$
f=\lim _{q \rightarrow+\infty}\left\{\sum_{i=-q}^{0} f * \tilde{\varphi}_{i} * \varphi_{i}-P_{q}\right\}+\sum_{i=1}^{\infty} f * \tilde{\varphi}_{i} * \varphi_{i}, \quad \tilde{\varphi}(x)=\overline{\varphi(-x)}
$$

we need a "discretization" on $f * \tilde{\varphi}_{i} * \varphi_{i}$. The discretization process is an extension of Shannon's formula, namely, Lemma 6.10 of [7]: Suppose that 
$g \in \mathcal{S}^{\prime}(\mathbb{R})$ and $h \in \mathcal{S}(\mathbb{R})$ with supp $\hat{g}, \operatorname{supp} \hat{h} \subset\left\{|\xi|:|\xi|<2^{\nu} \pi\right\}$ for some $\nu \in \mathbb{Z}$, then

$$
g * h(x)=\sum_{k \in \mathbb{Z}} 2^{-\nu} g\left(k 2^{-\nu}\right) h\left(x-k 2^{-\nu}\right)
$$

In fact, (3.4) is true when $\operatorname{supp} \hat{g}, \operatorname{supp} \hat{h} \subset \bigcup_{j=1}^{m} K_{j}$, where $\left\{K_{j}\right\}_{j=1}^{m}$ are closed intervals, and $\sum_{j=1}^{m}\left|K_{j}\right| \leq 2^{\nu+1} \pi$, where $\left|K_{j}\right|$ is the Lebesgue measure of $K_{j}$ (see pp. 63-64 of [7]).

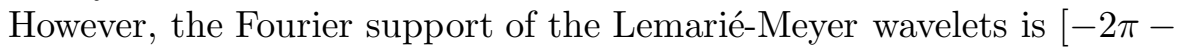
$\left.\epsilon^{\prime},-\pi+\epsilon\right] \cup\left[\pi-\epsilon, 2 \pi+\epsilon^{\prime}\right]$, for some $\epsilon, \epsilon^{\prime}>0$ satisfying $\epsilon+\epsilon^{\prime}<\pi$ (in this connection, see [8], Section 3.3, Theorem 3.1). If we consider the term corresponding to $i=0$ in (3.3); that is, $f * \tilde{\varphi} * \varphi$, and apply (3.4) to $g=f * \tilde{\varphi}$ and $h=\varphi$, then, we have to take $\nu=1$ (the Lebesgue measure of $\left[-2 \pi-\epsilon^{\prime},-\pi+\epsilon\right] \cup\left[\pi-\epsilon, 2 \pi+\epsilon^{\prime}\right]$ is $\left.2 \pi+2 \epsilon+2 \epsilon^{\prime}\right)$. The resulting expression,

$$
\begin{aligned}
(f * \tilde{\varphi} * \varphi)(x) & =\frac{1}{2} \sum_{k \in \mathbb{Z}}(f * \tilde{\varphi})(k / 2) \varphi(x-k / 2) \\
& =\frac{1}{2} \sum_{k \in \mathbb{Z}}\langle f(\cdot), \varphi(\cdot-k / 2)\rangle \varphi(x-k / 2),
\end{aligned}
$$

is not the wavelet expansion for the wavelet, $\varphi_{i, k}$, corresponding to $i=0$. Thus, Shannon's formula and the Littlewood-Paley analysis are useless in proving Theorem 3.1 for the Lemarié-Meyer wavelets.

Furthermore, the above argument also prohibits the use of Shannon's formula in proving Theorem 3.1 for any wavelet (no matter band-limited or not), $\varphi$, satisfying $|\operatorname{supp} \hat{\varphi}|>2 \pi$ (see [8], Section 2.2, Corollary 2.4).

For the MSF wavelet, $\varphi$, (that is, $|\operatorname{supp} \hat{\varphi}|=2 \pi)$ we find that $|\hat{\varphi}|=$ $\chi_{\operatorname{supp} \hat{\varphi}}$ (see [8], Section 2.2, Corollary 2.4). In this case, the discontinuity of $\hat{\varphi}$ forces a slow decay of $\varphi$ as $|x| \rightarrow \infty$. For example, consider Shannon's wavelet, $\hat{\varphi}(\xi)=e^{i \frac{\xi}{2}} \chi_{[-2 \pi,-\pi) \cup[\pi, 2 \pi)}(\xi)$. Then $\varphi(x)=-2 \frac{\sin 2 \pi x+\cos \pi x}{\pi(2 x+1)}$ (see [8], Section 2.2, Example C). Therefore, $\varphi$ does not belong to $\mathcal{S}(\mathbb{R})$ and, hence, the pairing, $\left\langle f, \varphi_{i, k}\right\rangle$, does not make sense if $f \in \mathcal{S}^{\prime}(\mathbb{R})$.

\subsection{Annihilator of wavelet system}

The following theorem shows that the annihilator for the wavelet system is equal to the set of polynomials.

TheORem 3.2. Let $\varphi \in \mathcal{S}(\mathbb{R})$ be a wavelet for $L^{2}(\mathbb{R})$, then we have

$$
\mathcal{P}(\mathbb{R})=\left\{f \in \mathcal{S}^{\prime}(\mathbb{R}):\left\langle f, \varphi_{i, k}\right\rangle=0, \forall i, k \in \mathbb{Z}\right\} .
$$


Proof. The inclusion, $\mathcal{P}(\mathbb{R}) \subseteq\left\{f \in \mathcal{S}^{\prime}(\mathbb{R}):\left\langle f, \varphi_{i, k}\right\rangle=0, \forall i, k \in \mathbb{Z}\right\}$, is guarantee by (1.8).

We apply Theorem 3.1 to prove the inclusion,

$$
\left\{f \in \mathcal{S}^{\prime}(\mathbb{R}):\left\langle f, \varphi_{i, k}\right\rangle=0, \forall i, k \in \mathbb{Z}\right\} \subseteq \mathcal{P}(\mathbb{R})
$$

For any $f \in \mathcal{S}^{\prime}(\mathbb{R})$ satisfying $\left\langle f, \varphi_{i, k}\right\rangle=0, \forall i, k \in \mathbb{Z}$, we find that there exists an integer $N>0$ and a family of polynomials, $P_{L, L^{\prime}, L^{\prime \prime}}$, such that

$$
f=\lim _{L, L^{\prime}, L^{\prime \prime} \rightarrow \infty} P_{L, L^{\prime}, L^{\prime \prime}} \quad \text { in } \mathcal{S}^{\prime}(\mathbb{R})
$$

The degrees of the polynomials, $P_{L, L^{\prime}, L^{\prime \prime}}$, are at most $N$ and $\mathcal{P}_{N}(\mathbb{R})$ is a finite dimensional subspace of $\mathcal{S}^{\prime}(\mathbb{R})$, hence, a closed subspace. Therefore, there exists a polynomial, $P$, of degree at most $N$ such that

$$
f=\lim _{L, L^{\prime}, L^{\prime \prime} \rightarrow \infty} P_{L, L^{\prime}, L^{\prime \prime}}=P,
$$

which is our desired result.

\subsection{Completeness of wavelet system}

Suppose that $B$ is a locally convex function space satisfying $\mathcal{S}(\mathbb{R}) \hookrightarrow$ $B \hookrightarrow \mathcal{S}^{\prime}(\mathbb{R})$ and $\mathcal{S}(\mathbb{R})$ is dense in $B$, with the Hahn-Banach theorem, Theorem 3.2 provides a condition that assert the completeness of the wavelet system, $\left\{\varphi_{i, k}\right\}_{i, k \in \mathbb{Z}}$, on $B$. The precise statement of this condition is given in the following theorem.

TheOREM 3.3. Let $\varphi \in \mathcal{S}(\mathbb{R})$ be a wavelet for $L^{2}(\mathbb{R})$. Suppose that $B$ is a locally convex function space such that $\mathcal{S}(\mathbb{R}) \hookrightarrow B \hookrightarrow \mathcal{S}^{\prime}(\mathbb{R})$ and $\mathcal{S}(\mathbb{R})$ is dense in $B$. The wavelet system, $\left\{\varphi_{i, k}\right\}_{i, k \in \mathbb{Z}}$, is complete in $B$ if and only if

$$
\mathcal{P}(\mathbb{R}) \cap B^{*}=\{0\} .
$$

Moreover, when $B^{*} \cap \mathcal{P}(\mathbb{R}) \neq\{0\},\left\{\varphi_{i, k}\right\}_{i, k \in \mathbb{Z}} \cup\{g\}$ is complete in $B$ if $g \in \mathcal{S}(\mathbb{R})$ satisfies

$$
\int x^{k} g(x) d x \neq 0, \quad \text { for all } k \in \mathbb{N} \text {. }
$$


Proof. As $\mathcal{S}(\mathbb{R})$ is dense in $B$, we have the embedding, $B^{*} \hookrightarrow \mathcal{S}^{\prime}(\mathbb{R})$. In view of $(3.5)$, we find that

$$
\left\{f \in B^{*}:\left\langle f, \varphi_{i, k}\right\rangle=0, \forall i, k \in \mathbb{Z}\right\}=B^{*} \cap \mathcal{P}(\mathbb{R}) .
$$

Thus, if $B^{*} \cap \mathcal{P}(\mathbb{R})=\{0\}$, then by the Hahn-Banach Theorem for locally convex space, the wavelet system, $\left\{\varphi_{i, k}\right\}_{i, k \in \mathbb{Z}}$, is complete in $B$. The second result follows from the observation,

$$
\begin{aligned}
& \left\{f \in B^{*}:\langle f, g\rangle=0 \text { and }\left\langle f, \varphi_{i, k}\right\rangle=0, \forall i, k \in \mathbb{Z}\right\} \\
& \quad=\{f \in \mathcal{P}(\mathbb{R}):\langle f, g\rangle=0\}=\{0\} .
\end{aligned}
$$

Note that the above result is optimal in the sense that if we remove any function from the sets, $\left\{\varphi_{i, k}\right\}_{i, k \in \mathbb{Z}}$ or $\left\{\varphi_{i, k}\right\}_{i, k \in \mathbb{Z}} \cup\{g\}$, then they are not complete in the corresponding function spaces. That is, using the terminology in [19], $\left\{\varphi_{i, k}\right\}_{i, k \in \mathbb{Z}}$ and $\left\{\varphi_{i, k}\right\}_{i, k \in \mathbb{Z}} \cup\{g\}$ are minimal (see Chapter I, Section 6 of [19]) in the corresponding function spaces.

The function, $g$, in Theorem 3.3 is easily constructed. Let $h \in \mathcal{S}(\mathbb{R})$ satisfy

$$
\int h(x) d x \neq 0, \quad \text { and } \quad \int x^{k} h(x) d x=0, \quad k \geq 1, k \in \mathbb{N} .
$$

Then, for any fixed $l \in \mathbb{R} \backslash\{0\}$, we can take $g(x)=h(x-l)$. For instance, if $\varphi$ is the Meyer wavelet and $\Phi$ is the corresponding scaling function, then we know that $\Phi$ satisfies (3.7). Thus, for any fixed $l \in \mathbb{R} \backslash\{0\}$, the system, $\left\{\varphi_{i, k}(x)\right\}_{i, k \in \mathbb{Z}} \cup\{\Phi(x-l)\}$, is complete in any function space $B$ satisfying the conditions in Theorem 3.3. In particular, $\left\{\varphi_{i, k}(x)\right\}_{i, k \in \mathbb{Z}} \cup\{\Phi(x-l)\}$ is complete in $L^{1}(\mathbb{R})$. In Section 5.1, we show that we can relax the condition, $l \in \mathbb{R} \backslash\{0\}$, to, $l \in \mathbb{R}$, for the completeness of the wavelet system in $L^{1}(\mathbb{R})$.

Using the idea for proving Theorem 3.3, we can show that if $B$ is a locally convex function space satisfying $\mathcal{S}_{0}(\mathbb{R})=\left\{f \in \mathcal{S}(\mathbb{R}): \int x^{\gamma} f(x) d x=\right.$ $0, \gamma \in \mathbb{N}\} \hookrightarrow B \hookrightarrow \mathcal{S}^{\prime}(\mathbb{R}) / \mathcal{P}(\mathbb{R})$ and $\mathcal{S}_{0}(\mathbb{R})$ is dense in $B$, then $\left\{\varphi_{i, k}\right\}_{i, k \in \mathbb{Z}}$ is a complete system in $B$.

\section{$\S 4$. Proof of Theorem 2.1}

\subsection{Preliminary results}

For a $r$-times differentiable function, $\varphi$, defined on $\mathbb{R}^{n}$, the $\gamma^{\text {th }}$-order partial derivative of $\varphi, \gamma \in \mathbb{N}^{n}$ and $|\gamma| \leq r$, is denoted by $\partial^{\gamma} \varphi$ or $\frac{\partial^{\gamma} \varphi}{\partial x^{\gamma}}$. 
Definition 4.1. Given a fixed $\epsilon>0$, for any $\alpha>0$, we denote $\mathcal{C}_{\alpha}\left(\mathbb{R}^{n}\right)$ to be the class of functions, $\varphi(x)$, satisfying

$$
\|\varphi\|_{\alpha *}=\sup _{0 \leq|\gamma| \leq[\alpha]} \sup _{x \in \mathbb{R}^{n}}\left|(1+|x|)^{\alpha+n+\epsilon} \partial^{\gamma} \varphi(x)\right|<\infty,
$$

where $\gamma \in \mathbb{N}^{n}$ and $[\alpha]$ is the integer part of $\alpha$; and

$$
\|\varphi\|_{\alpha * *}=\sup _{\substack{|\gamma|=[\alpha] \\ \gamma \in \mathbb{N}^{n}}} \sup _{\substack{x, y \in \mathbb{R}^{n}, x \neq y \\|z| \leq|x-y|}}(1+|x-z|)^{\alpha+n+\epsilon} \frac{\left|\partial^{\gamma} \varphi(x)-\partial^{\gamma} \varphi(y)\right|}{|x-y|^{\alpha-[\alpha]}}<\infty
$$

if $\alpha>[\alpha]$. Let the norm on $\mathcal{C}_{\alpha}\left(\mathbb{R}^{n}\right)$ be $\|\varphi\|_{\mathcal{C}_{\alpha}}=\max \left\{\|\varphi\|_{\alpha *},\|\varphi\|_{\alpha * *}\right\}$. Let $\mathcal{S}_{\alpha}\left(\mathbb{R}^{n}\right)$ consist of functions satisfying

$$
\|\varphi\|_{\alpha \sharp}=\sup _{0 \leq|\gamma| \leq[\alpha]} \sup _{x \in \mathbb{R}^{n}}\left|(1+|x|)^{\alpha+n} \partial^{\gamma} \varphi(x)\right|<\infty,
$$

where $\gamma \in \mathbb{N}^{n} ;$ and

$$
\|\varphi\|_{\alpha \sharp \sharp}=\sup _{\substack{|\gamma|=[\alpha] \\ \gamma \in \mathbb{N}^{n}}} \sup _{\substack{x, y \in \mathbb{R}^{n}, x \neq y \\|z| \leq|x-y|}}(1+|x-z|)^{\alpha+n} \frac{\left|\partial^{\gamma} \varphi(x)-\partial^{\gamma} \varphi(y)\right|}{|x-y|^{\alpha-[\alpha]}}<\infty
$$

if $\alpha>[\alpha]$. Let the norm on $\mathcal{S}_{\alpha}\left(\mathbb{R}^{n}\right)$ be $\|\varphi\|_{\mathcal{S}_{\alpha}}=\max \left\{\|\varphi\|_{\alpha \sharp},\|\varphi\|_{\alpha \sharp \sharp}\right\}$.

Remark 4.1. In Lemma 7.1, we will apply Lemma B.1 of [6]. Therefore, we need an extra $\epsilon$-decay for the definition of $\mathcal{C}_{\alpha}\left(\mathbb{R}^{n}\right)$ in order to fulfill the conditions in Lemma B.1 of [6].

It is easy to see that $\mathcal{C}_{\alpha}\left(\mathbb{R}^{n}\right)$ and $\mathcal{S}_{\alpha}\left(\mathbb{R}^{n}\right)$ are Banach spaces. In addition, we have $\mathcal{S}\left(\mathbb{R}^{n}\right)=\bigcap_{\alpha>0} \mathcal{S}_{\alpha}\left(\mathbb{R}^{n}\right)$. The space of Schwartz functions, $\mathcal{S}\left(\mathbb{R}^{n}\right)$, is a Fréchet space and its topology is generated by the semi-norms, $\|\cdot\| \mathcal{S}_{\alpha}$, $\alpha>0$. Hence, the embedding, $\mathcal{S}\left(\mathbb{R}^{n}\right) \hookrightarrow \mathcal{S}_{\alpha}\left(\mathbb{R}^{n}\right)$, is continuous for any $\alpha>0$.

Let $\mathcal{S}_{\alpha}\left(\mathbb{R}^{n}\right)^{*}$ denote the dual space of $\mathcal{S}_{\alpha}\left(\mathbb{R}^{n}\right)$. For any $f \in \mathcal{S}^{\prime}\left(\mathbb{R}^{n}\right)$, the continuity of $f$ in $\mathcal{S}\left(\mathbb{R}^{n}\right)$ means that there exist $\alpha>0$ and a constant $C>0$, such that $|\langle f, \psi\rangle| \leq C\|\psi\|_{\mathcal{S}_{\alpha}\left(\mathbb{R}^{n}\right)}, \forall \psi \in \mathcal{S}\left(\mathbb{R}^{n}\right)$. Therefore, by the Hahn-Banach Theorem, $f$ can be extended to a distribution in $\mathcal{S}_{\alpha}\left(\mathbb{R}^{n}\right)$. That is,

$$
f \in \mathcal{S}_{\alpha}\left(\mathbb{R}^{n}\right)^{*}
$$


Moreover, we have $\mathcal{S}^{\prime}\left(\mathbb{R}^{n}\right)=\bigcup_{\alpha>0} \mathcal{S}_{\alpha}\left(\mathbb{R}^{n}\right)^{*}$.

For any given $f \in \mathcal{S}^{\prime}\left(\mathbb{R}^{n}\right)$, we call the infimum of those $\alpha$ such that (4.5) holds the order of $f \in \mathcal{S}^{\prime}\left(\mathbb{R}^{n}\right)$. It is easy to see that if $f \in \mathcal{S}^{\prime}\left(\mathbb{R}^{n}\right)$ is of order $\omega$, then, for any $\eta>\omega$, there exists $C>0$ such that $|\langle f, \psi\rangle| \leq C\|\psi\|_{\mathcal{C}_{\eta}\left(\mathbb{R}^{n}\right)}$, $\forall \psi \in \mathcal{C}_{\eta}\left(\mathbb{R}^{n}\right)$. Hence, $f$ can be extended to be a distribution on $\mathcal{C}_{\eta}\left(\mathbb{R}^{n}\right)$ with any $\eta>\omega$.

For any $\alpha>0$, let $\mathcal{C}_{\alpha}\left(\mathbb{R}^{n}\right)^{*}$ denote the dual space of $\mathcal{C}_{\alpha}\left(\mathbb{R}^{n}\right)$. Notice that if $f \in \mathcal{C}_{\alpha}\left(\mathbb{R}^{n}\right)^{*}$ and $g \in \mathcal{C}_{\alpha}\left(\mathbb{R}^{n}\right)$, then $\langle f, g\rangle$ is the pairing between $\mathcal{C}_{\alpha}\left(\mathbb{R}^{n}\right)^{*}$ and $\mathcal{C}_{\alpha}\left(\mathbb{R}^{n}\right)$ (we use this pairing in Theorem 4.1). Furthermore, the embedding, $\mathcal{C}_{\alpha}\left(\mathbb{R}^{n}\right)^{*} \hookrightarrow \mathcal{S}^{\prime}\left(\mathbb{R}^{n}\right)$, is continuous.

Theorem 2.1 relies on the following result, Theorem 4.1. Theorem 4.1 has its own independent interest. It provides the condition for which the frame expansion of Schwartz function converges in the topology of $\mathcal{S}\left(\mathbb{R}^{n}\right)$. In addition, it shows that, for the convergence of frame expansion of $g \in$ $\mathcal{C}_{\alpha}\left(\mathbb{R}^{n}\right)$, the rate of convergence depends on the difference between the smoothness of the frame and the smoothness of the function spaces used to measure the "error" between $g$ and its truncated frame expansion.

Theorem 4.1 is closely related to the convergence of wavelet expansion for Lipschitz functions and two-microlocal functions (for the definitions and details of these function spaces, see [8], Section 6.7, and [16], Chapter 3, Definition 3.1 and Theorem 3.6, respectively). While they are neither stronger nor weaker, they represent different results under different assumptions.

Theorem 4.1. (Convergence theorem for frame expansion of Schwartz functions) Let $\left\{\varphi_{Q}\right\}_{Q \in \mathcal{Q}}$ be a frame on $L^{2}\left(\mathbb{R}^{n}\right)$ and $\left\{\psi_{Q}\right\}_{Q \in \mathcal{Q}}$ be its dual frame. Let $\alpha, \beta>0$ with $\alpha>[\alpha], \alpha>\beta+\epsilon$ (the $\epsilon$ in Definition 2.1).

(1) Suppose that $\left\{\varphi_{Q}\right\}_{Q},\left\{\psi_{Q}\right\}_{Q} \in \mathcal{M}_{\alpha}\left(\mathbb{R}^{n}\right)$. The truncated frame expansion of $g \in \mathcal{C}_{\alpha}\left(\mathbb{R}^{n}\right)$,

$$
\sum_{i=-L^{\prime}}^{L} \sum_{|k| \leq \delta(i) 2^{L^{\prime \prime}}}\left\langle g, \varphi_{Q_{i, k}}\right\rangle \psi_{Q_{i, k}}
$$

converges to $g$ in $\mathcal{C}_{\beta}\left(\mathbb{R}^{n}\right)$ as $L, L^{\prime}$ and $L^{\prime \prime}$ go to infinity if

$$
\int_{\mathbb{R}^{n}} x^{\lambda} g(x) d x=0 \quad \text { for }|\lambda| \leq[\alpha], \lambda \in \mathbb{N}^{n} .
$$


Moreover, there is a constant, $C>0$, independent of $L, L^{\prime}, L^{\prime \prime}$ such that, for any $g \in \mathcal{C}_{\alpha}\left(\mathbb{R}^{n}\right)$ satisfying (4.7),

$$
\begin{aligned}
& \left\|g-\sum_{i=-L^{\prime}}^{L} \sum_{|k| \leq \delta(i) 2^{L^{\prime \prime}}}\left\langle g, \varphi_{Q_{i, k}}\right\rangle \psi_{Q_{i, k}}\right\|_{\mathcal{C}_{\beta}} \\
& \quad \leq C\|g\|_{\mathcal{C}_{\alpha}}\left(2^{(-L-1)(\alpha-\beta)}+2^{\left(-L^{\prime}-1\right)(\alpha-\beta-\epsilon)}+2^{-L^{\prime \prime}(\alpha-\beta)}\right) .
\end{aligned}
$$

(2) Suppose that $\left\{\varphi_{Q}\right\}_{Q},\left\{\psi_{Q}\right\}_{Q} \in \bigcap_{\alpha} \mathcal{M}_{\alpha}\left(\mathbb{R}^{n}\right)$. The truncated frame expansion of $g \in \mathcal{S}\left(\mathbb{R}^{n}\right)$,

$$
\sum_{i=-L^{\prime}}^{L} \sum_{|k| \leq \delta(i) 2^{L^{\prime \prime}}}\left\langle g, \varphi_{Q_{i, k}}\right\rangle \psi_{Q_{i, k}}
$$

converges to $g$ in $\mathcal{S}\left(\mathbb{R}^{n}\right)$ as $L, L^{\prime}, L^{\prime \prime} \rightarrow \infty$ if and only if

$$
\int_{\mathbb{R}^{n}} x^{\lambda} g(x) d x=0 \quad \text { for all } \lambda \in \mathbb{N}^{n} .
$$

That is, for any $g \in \mathcal{S}_{0}\left(\mathbb{R}^{n}\right)$, the truncated frame expansion, (4.9), converges to $g$ in $\mathcal{S}\left(\mathbb{R}^{n}\right)$.

We prove the above Theorem by estimating the inner product of function in $\mathcal{C}_{\alpha}\left(\mathbb{R}^{n}\right)$ and frame in $\mathcal{M}_{\alpha}\left(\mathbb{R}^{n}\right)$. The estimate comes from Lemma B.1 of [6]. The proof of Theorem 4.1 is provided in Section 7 .

The implication, (4.9) $\Rightarrow(4.10)$, is easy because, for any fixed $\lambda \in$ $\mathbb{N}^{n}, f(x)=x^{\lambda}$ is a Schwartz distribution and the dual frame, $\left\{\psi_{Q}\right\}_{Q \in \mathcal{Q}} \in$ $\bigcap_{\alpha} \mathcal{M}_{\alpha}\left(\mathbb{R}^{n}\right)$, satisfies

$$
\int_{\mathbb{R}^{n}} x^{\lambda} \psi_{Q}(x) d x=0 \quad \text { for all } \lambda \in \mathbb{N}^{n} \text { and } Q \in \mathcal{Q} .
$$

The reserve implication is a straightforward consequence of $\mathcal{S}\left(\mathbb{R}^{n}\right)=$ $\bigcap_{\alpha>0} \mathcal{C}_{\alpha}\left(\mathbb{R}^{n}\right)$ and the first part of Theorem 4.1. Similarly, if $[\alpha]=[\beta]$, then (4.6) holds if and only if $g$ satisfies (4.7).

\subsection{Proof of Theorem 2.1}

With the above preparations, we are now ready to prove Theorem 2.1. We are going to prove that for any $f \in \mathcal{S}^{\prime}\left(\mathbb{R}^{n}\right)$, there exist an integer $N \geq 0$ 
and a sequence of floating polynomials $P_{L, L^{\prime}, L^{\prime \prime}}(x)$ of degrees less than or equal to $N$ such that, for any $g \in \mathcal{S}\left(\mathbb{R}^{n}\right)$,

$$
\lim _{L, L^{\prime}, L^{\prime \prime} \rightarrow \infty}\left\{\sum_{i=-L^{\prime}}^{L} \sum_{|k| \leq \delta(i) 2^{L^{\prime \prime}}}\left\langle f, \psi_{Q_{i, k}}\right\rangle\left\langle\varphi_{Q_{i, k}}, g\right\rangle-\left\langle P_{L, L^{\prime}, L^{\prime \prime}}, g\right\rangle\right\}=\langle f, g\rangle .
$$

Since $\mathcal{S}^{\prime}\left(\mathbb{R}^{n}\right)$ is endowed with the weak-star topology inherited from $\mathcal{S}\left(\mathbb{R}^{n}\right)$, (4.12) shows that

$$
\lim _{L, L^{\prime}, L^{\prime \prime} \rightarrow \infty}\left\{\sum_{i=-L^{\prime}}^{L} \sum_{|k| \leq \delta(i) 2^{L^{\prime \prime}}}\left\langle f, \psi_{Q_{i, k}}\right\rangle \varphi_{Q_{i, k}}-P_{L, L^{\prime}, L^{\prime \prime}}\right\}=f
$$

in the topology of $\mathcal{S}^{\prime}\left(\mathbb{R}^{n}\right)$.

Suppose that $f$ is a distribution in $\mathcal{S}^{\prime}\left(\mathbb{R}^{n}\right)$ of order $\omega$. We consider the family of function spaces, $\mathcal{C}_{\alpha}\left(\mathbb{R}^{n}\right)$, with $\epsilon=([\omega]+1-\omega) / 2$.

For any $\lambda \in \mathbb{N}^{n}$ with $0 \leq|\lambda| \leq[\omega]$, let $\theta_{\lambda} \in \mathcal{S}\left(\mathbb{R}^{n}\right)$ satisfy

$$
\int_{\mathbb{R}^{n}} x^{\gamma} \theta_{\lambda}(x) d x=\delta_{\gamma \lambda}=\left\{\begin{array}{ll}
1, & \gamma=\lambda \\
0, & \gamma \neq \lambda,
\end{array} \text { for } \gamma \in \mathbb{N}^{n} \text { and } 0 \leq|\gamma| \leq[\omega] .\right.
$$

For any $g \in \mathcal{S}\left(\mathbb{R}^{n}\right)$, define $G$ by

$$
G(x)=g(x)-\sum_{0 \leq|\lambda| \leq[\omega]} c_{\lambda} \theta_{\lambda}(x)
$$

where $c_{\lambda}=\int_{\mathbb{R}^{n}} x^{\lambda} g(x) d x$. It is obvious that $G \in \mathcal{S}\left(\mathbb{R}^{n}\right)$ and

$$
\int_{\mathbb{R}^{n}} x^{\lambda} G(x) d x=0, \quad \text { if } \lambda \in \mathbb{N}^{n} \text { and } 0 \leq|\lambda| \leq[\omega] .
$$

Thus, by the first part of Theorem 4.1, we assert that

$$
\lim _{L, L^{\prime}, L^{\prime \prime} \rightarrow \infty} \sum_{i=-L^{\prime}}^{L} \sum_{|k| \leq \delta(i) 2^{L^{\prime \prime}}}\left\langle G, \varphi_{Q_{i, k}}\right\rangle \psi_{Q_{i, k}}=G \quad \text { in } \mathcal{C}_{\eta}\left(\mathbb{R}^{n}\right),
$$

for any $\eta>\omega$ satisfying $[\eta]=[\omega]$ and $[\omega]+1>\eta+\epsilon$.

Since $f \in \mathcal{S}^{\prime}\left(\mathbb{R}^{n}\right)$ is of order $\omega$ and $\eta>\omega$, this allows us to apply $f$ on both sides of (4.15). We obtain

$$
\lim _{L, L^{\prime}, L^{\prime \prime} \rightarrow \infty} \sum_{i=-L^{\prime}}^{L} \sum_{|k| \leq \delta(i) 2^{L^{\prime \prime}}}\left\langle\varphi_{Q_{i, k}}, G\right\rangle\left\langle f, \psi_{Q_{i, k}}\right\rangle=\langle f, G\rangle
$$


because $\mathcal{S}^{\prime}\left(\mathbb{R}^{n}\right)$ is endowed with the antilinear structure mentioned at the beginning of Section 1.

By using the definition of $G,(4.14)$, and the fact that $\bar{c}_{\lambda}=\int_{\mathbb{R}^{n}} x^{\lambda} \overline{g(x)} d x$ $=\left\langle x^{\lambda}, g\right\rangle$, we have

$$
\begin{aligned}
& \lim _{L, L^{\prime}, L^{\prime \prime} \rightarrow \infty} \sum_{i=-L^{\prime}}^{L} \sum_{|k| \leq \delta(i) 2^{L^{\prime \prime}}}\left\{\left\langle\varphi_{Q_{i, k}}, g\right\rangle\left\langle f, \psi_{Q_{i, k}}\right\rangle\right. \\
& \left.-\sum_{0 \leq|\lambda| \leq[\omega]}\left\langle x^{\lambda}, g\right\rangle\left\langle\varphi_{Q_{i, k}}, \theta_{\lambda}\right\rangle\left\langle f, \psi_{Q_{i, k}}\right\rangle\right\} \\
& =\langle f, g\rangle-\sum_{0 \leq|\lambda| \leq[\omega]}\left\langle x^{\lambda}, g\right\rangle\left\langle f, \theta_{\lambda}\right\rangle .
\end{aligned}
$$

If we write

$$
P_{L, L^{\prime}, L^{\prime \prime}}(x)=-\sum_{0 \leq|\lambda| \leq[\omega]}\left(\left\langle f, \theta_{\lambda}\right\rangle-\sum_{i=-L^{\prime}}^{L} \sum_{|k| \leq \delta(i) 2^{L^{\prime \prime}}}\left\langle\varphi_{Q_{i, k}}, \theta_{\lambda}\right\rangle\left\langle f, \psi_{Q_{i, k}}\right\rangle\right) x^{\lambda}
$$

then we obtain

$$
\lim _{L, L^{\prime}, L^{\prime \prime} \rightarrow \infty}\left\{\sum_{i=-L^{\prime}}^{L} \sum_{|k| \leq \delta(i) 2^{L^{\prime \prime}}}\left\langle f, \psi_{Q_{i, k}}\right\rangle\left\langle\varphi_{Q_{i, k}}, g\right\rangle-\left\langle P_{L, L^{\prime}, L^{\prime \prime}}, g\right\rangle\right\}=\langle f, g\rangle,
$$

which is our desired result, (4.12). Hence, we prove Theorem 2.1 with $N=[\omega]$.

Since $\theta_{\lambda}$ satisfies (4.13) and any frame belonging to $\bigcap \mathcal{M}_{\alpha}\left(\mathbb{R}^{n}\right)$ satisfies (4.11), using our formula for $P_{L, L^{\prime}, L^{\prime \prime}}(x)$, the floating polynomials associated with $f(x)=x^{\lambda}, \lambda \in \mathbb{N}^{n}$ and $0 \leq|\lambda| \leq[\omega]$, are equal to $P_{L, L^{\prime}, L^{\prime \prime}}(x)=-x^{\lambda}$. In addition, by considering the Schwartz distribution, $f(x)=x^{\lambda}$, with $\lambda=$ $[\omega]$, we find that Theorem 2.1 is not valid if we take $N<[\omega]$.

The proof of Theorem 2.1 is sufficient to justify the introduction of the non-integer order for the family of function spaces, $\mathcal{C}_{\alpha}\left(\mathbb{R}^{n}\right)$. If we do not have $\mathcal{C}_{\alpha}\left(\mathbb{R}^{n}\right)$ when $\alpha \notin \mathbb{N}$, we cannot obtain the optimal result for the degree of the floating polynomials.

\section{$\S 5$. Further results}

\subsection{Frames belonging to $\mathcal{M}_{\alpha}\left(\mathbb{R}^{n}\right)$}

In this section, we investigate the convergence of frame expansion with the frame, $\left\{\varphi_{Q}\right\}_{Q \in \mathcal{Q}}$ and its dual frame $\left\{\psi_{Q}\right\}_{Q \in \mathcal{Q}}$, only satisfying $\left\{\varphi_{Q}\right\}_{Q}$, 
$\left\{\psi_{Q}\right\}_{Q} \in \mathcal{M}_{\alpha}\left(\mathbb{R}^{n}\right)$. In this case, $\left\langle f, \psi_{Q}\right\rangle$ is not well defined if $f \in$ $\mathcal{S}^{\prime}\left(\mathbb{R}^{n}\right) \backslash \mathcal{C}_{\alpha}\left(\mathbb{R}^{n}\right)^{*}$. Therefore, we can obtain a reasonable result only if we consider distributions of order less than $\alpha$.

Theorem 5.1. Given $\epsilon>0$ (the $\epsilon$ in Definition 4.1) and $\alpha>[\alpha]$, if $\left\{\varphi_{Q}\right\}_{Q \in \mathcal{Q}} \in \mathcal{M}_{\alpha}\left(\mathbb{R}^{n}\right)$ is a frame on $L^{2}\left(\mathbb{R}^{n}\right)$ and $\left\{\psi_{Q}\right\}_{Q \in \mathcal{Q}} \in \mathcal{M}_{\alpha}\left(\mathbb{R}^{n}\right)$ is its dual frame, then for any $f \in \mathcal{C}_{\beta}\left(\mathbb{R}^{n}\right)^{*}$ with $\alpha>\beta+\epsilon$, there exists a sequence of floating polynomials,

$$
P_{L, L^{\prime}, L^{\prime \prime}}(x)=-\sum_{0 \leq|\lambda| \leq[\alpha]}\left(\left\langle f, \theta_{\lambda}\right\rangle-\sum_{i=-L^{\prime}}^{L} \sum_{|k| \leq \delta(i) L^{L^{\prime \prime}}}\left\langle\varphi_{Q_{i, k}}, \theta_{\lambda}\right\rangle\left\langle f, \psi_{Q_{i, k}}\right\rangle\right) x^{\lambda},
$$

where $\lambda \in \mathbb{N}^{n}$ and $\theta_{\lambda} \in \mathcal{C}_{\alpha}\left(\mathbb{R}^{n}\right)$ satisfies

$$
\int_{\mathbb{R}^{n}} x^{\gamma} \theta_{\lambda}(x) d x=\delta_{\gamma \lambda} \quad \text { for } \gamma \in \mathbb{N}^{n} \text { and } 0 \leq|\gamma| \leq[\alpha],
$$

such that

$$
\lim _{L, L^{\prime}, L^{\prime \prime} \rightarrow \infty}\left\{\sum_{i=-L^{\prime}}^{L} \sum_{|k| \leq \delta(i) 2^{L^{\prime \prime}}}\left\langle f, \psi_{Q_{i, k}}\right\rangle \varphi_{Q_{i, k}}-P_{L, L^{\prime}, L^{\prime \prime}}\right\}=f
$$

in $\mathcal{C}_{\alpha}\left(\mathbb{R}^{n}\right)^{*}$ and, hence, in $\mathcal{S}^{\prime}\left(\mathbb{R}^{n}\right)$. Furthermore, there is a constant $C>0$ independent of $L, L^{\prime}, L^{\prime \prime}$ and $f \in \mathcal{C}_{\beta}\left(\mathbb{R}^{n}\right)^{*}$ such that

$$
\begin{aligned}
& \left\|f-\sum_{i=-L^{\prime}}^{L} \sum_{|k| \leq \delta(i) 2^{L^{\prime \prime}}}\left\langle f, \psi_{Q_{i, k}}\right\rangle \varphi_{Q_{i, k}}+P_{L, L^{\prime}, L^{\prime \prime}}\right\|_{\mathcal{C}_{\alpha}^{*}} \\
& \leq C\|f\|_{\mathcal{C}_{\beta}^{*}}\left(2^{(-L-1)(\alpha-\beta)}+2^{\left(-L^{\prime}-1\right)(\alpha-\beta-\epsilon)}+2^{-L^{\prime \prime}(\alpha-\beta)}\right) .
\end{aligned}
$$

Proof. The proof for Theorem 2.1 carries over to this case. We therefore only need to prove (5.3).

First of all, we have

$$
f-\sum_{i=-L^{\prime}}^{L} \sum_{|k| \leq \delta(i) 2^{L^{\prime \prime}}}\left\langle f, \psi_{Q_{i, k}}\right\rangle \varphi_{Q_{i, k}}+P_{L, L^{\prime}, L^{\prime \prime}} \in \mathcal{C}_{\alpha}\left(\mathbb{R}^{n}\right)^{*}
$$

because $f \in \mathcal{C}_{\beta}\left(\mathbb{R}^{n}\right)^{*} \hookrightarrow \mathcal{C}_{\alpha}\left(\mathbb{R}^{n}\right)^{*}$ and $P_{L, L^{\prime}, L^{\prime \prime}} \in \mathcal{C}_{\alpha}\left(\mathbb{R}^{n}\right)^{*}$. 
Inequality (5.3) is established by considering

$$
\begin{aligned}
\| f & -\sum_{i=-L^{\prime}}^{L} \sum_{|k| \leq \delta(i) 2^{L^{\prime \prime}}}\left\langle f, \psi_{Q_{i, k}}\right\rangle \varphi_{Q_{i, k}}+P_{L, L^{\prime}, L^{\prime \prime}} \|_{\mathcal{C}_{\alpha}^{*}} \\
= & \sup _{\|g\|_{\mathcal{C}_{\alpha}=1}}\left|\left\langle f-\sum_{i=-L^{\prime}} \sum_{|k| \leq \delta(i) 2^{L^{\prime \prime}}}\left\langle f, \psi_{Q_{i, k}}\right\rangle \varphi_{Q_{i, k}}+P_{L, L^{\prime}, L^{\prime \prime}}, g\right\rangle\right| \\
= & \sup _{\|g\|_{\mathcal{C}_{\alpha}=1}}\left|\langle f, g\rangle-\sum_{i=-L^{\prime}}^{L} \sum_{|k| \leq \delta(i) 2^{L^{\prime \prime}}}\left\langle f, \psi_{Q_{i, k}}\right\rangle\left\langle\varphi_{Q_{i, k}}, g\right\rangle+\left\langle P_{L, L^{\prime}, L^{\prime \prime}}, g\right\rangle\right| .
\end{aligned}
$$

By the definition of $P_{L, L^{\prime}, L^{\prime \prime}}(x)$, we find that

$$
\begin{aligned}
& \left\|f-\sum_{i=-L^{\prime}}^{L} \sum_{|k| \leq \delta(i) 2^{L^{\prime \prime}}}\left\langle f, \psi_{Q_{i, k}}\right\rangle \varphi_{Q_{i, k}}+P_{L, L^{\prime}, L^{\prime \prime}}\right\|_{\mathcal{C}_{\alpha}^{*}} \\
& =\sup _{\|g\|_{\mathcal{C}_{\alpha}=1}}\left|\langle f, G\rangle-\sum_{i=-L^{\prime}}^{L} \sum_{|k| \leq \delta(i) 2^{L^{\prime \prime}}}\left\langle\varphi_{Q_{i, k}}, G\right\rangle\left\langle f, \psi_{Q_{i, k}}\right\rangle\right|,
\end{aligned}
$$

where, for any $g \in \mathcal{C}_{\alpha}\left(\mathbb{R}^{n}\right), G$ is given by (4.13) and (4.14) with $\omega$ replaced by $\alpha$.

Therefore,

$$
\begin{aligned}
& \left\|f-\sum_{i=-L^{\prime}}^{L} \sum_{|k| \leq \delta(i) 2^{L^{\prime \prime}}}\left\langle f, \psi_{Q_{i, k}}\right\rangle \varphi_{Q_{i, k}}+P_{L, L^{\prime}, L^{\prime \prime}}\right\|_{\mathcal{C}_{\alpha}^{*}} \\
& \leq \sup _{\|g\|_{\mathcal{C}_{\alpha}=1}}\|f\|_{\mathcal{C}_{\beta}^{*}}\left\|G-\sum_{i=-L^{\prime}}^{L} \sum_{|k| \leq \delta(i) 2^{L^{\prime \prime}}}\left\langle G, \varphi_{Q_{i, k}}\right\rangle \psi_{Q_{i, k}}\right\|_{\mathcal{C}_{\beta}} .
\end{aligned}
$$

Since $G \in \mathcal{C}_{\alpha}\left(\mathbb{R}^{n}\right)$ satisfies the conditions in Theorem 4.1 and there exists $C>0$ such that $\|G\|_{\mathcal{C}_{\alpha}} \leq C\|g\|_{\mathcal{C}_{\alpha}}$, by applying (5.11), we have

$$
\begin{aligned}
& \left\|f-\sum_{i=-L^{\prime}}^{L} \sum_{|k| \leq \delta(i) 2^{L^{\prime \prime}}}\left\langle f, \psi_{Q_{i, k}}\right\rangle \varphi_{Q_{i, k}}+P_{L, L^{\prime}, L^{\prime \prime}}\right\|_{\mathcal{C}_{\alpha}^{*}} \\
& \leq C\|f\|_{\mathcal{C}_{\beta}^{*}} \sup _{\|g\|_{\mathcal{C}_{\alpha}=1}}\|g\|_{\mathcal{C}_{\alpha}}\left(2^{(-L-1)(\alpha-\beta)}+2^{\left(-L^{\prime}-1\right)(\alpha-\beta-\epsilon)}+2^{-L^{\prime \prime}(\alpha-\beta)}\right) \\
& \leq C\|f\|_{\mathcal{C}_{\beta}^{*}}\left(2^{(-L-1)(\alpha-\beta)}+2^{\left(-L^{\prime}-1\right)(\alpha-\beta-\epsilon)}+2^{-L^{\prime \prime}(\alpha-\beta)}\right) \cdot
\end{aligned}
$$


The Daubechies wavelets do not satisfy the conditions in Theorem 2.1; therefore, we need Theorem 5.1 for the Daubechies wavelets.

The degree of the floating polynomials in Theorem 2.1 is equal to the integral part of the order of the Schwartz distribution, $[\omega]$, while the degree of the floating polynomials in Theorem 5.1 is defined by the smoothness of the frame functions, $[\alpha]$. This may give a wrong impression that Theorem 5.1 is inconsistent with Theorem 2.1. In fact, they are complementary. Theorem 2.1 is valid for any $f \in \mathcal{S}^{\prime}(\mathbb{R})$ but Theorem 5.1 only applies to $f \in \mathcal{C}_{\beta}(\mathbb{R})^{*} \subsetneq \mathcal{S}^{\prime}(\mathbb{R})$. On the other hand, the estimate, (5.3), is stronger than the convergence, (2.4).

\subsection{One-dimensional wavelets in $\mathcal{C}_{\alpha}(\mathbb{R})$}

With Theorem 5.1, we can obtain the following analogue of Theorem 3.2 .

Theorem 5.2. Let $\beta>[\beta] \geq 0$ and $\eta>\beta$. Suppose that $\varphi$ is a wavelet belonging to $\mathcal{S}_{\eta}(\mathbb{R})$, then we have

$$
\mathcal{P}_{[\beta]}(\mathbb{R})=\left\{f \in \mathcal{S}_{\beta}(\mathbb{R})^{*}:\left\langle f, \varphi_{i, k}\right\rangle=0, i, k \in \mathbb{Z}\right\} .
$$

Proof. Let us consider the family of function spaces $\left\{\mathcal{C}_{\alpha}(\mathbb{R})\right\}_{\alpha>0}$ with $\epsilon<(\eta-\beta) / 4$. Without loss of generality, we assume that $\eta-\epsilon>[\eta-\epsilon]=[\eta]$. Then, we have $\varphi \in \mathcal{S}_{\eta}(\mathbb{R}) \subset \mathcal{C}_{\eta-\epsilon}(\mathbb{R})$. We are allowed to apply Theorem 5.1 with $\alpha=\eta-\epsilon$. Thus, for any $f \in \mathcal{S}_{\beta}(\mathbb{R})^{*} \subset \mathcal{C}_{\beta}(\mathbb{R})^{*}$ satisfying $\left\langle f, \varphi_{i, k}\right\rangle=0$, $\forall i, k \in \mathbb{Z}$, there exists a polynomial, $P$, of degree at most $[\eta]$ such that $f=P$. Moreover, since $f \in \mathcal{S}_{\beta}(\mathbb{R})^{*}, f$ is a polynomial of degree at most $[\beta]$. The result in (1.8) asserts the reserve inclusion, $\mathcal{P}_{[\beta]}(\mathbb{R}) \subseteq\left\{f \in \mathcal{S}_{\beta}(\mathbb{R})^{*}\right.$ : $\left.\left\langle f, \varphi_{i, k}\right\rangle=0, i, k \in \mathbb{Z}\right\}$.

By using Theorem 5.2, we extend the results in Theorem 3.3 as follow:

Theorem 5.3. Let $\beta>[\beta] \geq 0$ and $\eta>\beta$. Let $\varphi \in \mathcal{S}_{\eta}(\mathbb{R})$ be a wavelet for $L^{2}(\mathbb{R})$ and $B$ be a locally convex function space such that $\mathcal{S}_{\beta}(\mathbb{R}) \hookrightarrow$ $B \hookrightarrow \mathcal{S}_{\beta}(\mathbb{R})^{*}$ and $\mathcal{S}_{\beta}(\mathbb{R})$ is dense in $B$. The wavelet system, $\left\{\varphi_{i, k}\right\}_{i, k \in \mathbb{Z}}$, is complete in $B$ if and only if

$$
\mathcal{P}_{[\beta]}(\mathbb{R}) \cap B^{*}=\{0\} .
$$

Moreover, when $\mathcal{P}_{[\beta]}(\mathbb{R}) \cap B^{*} \neq\{0\}$, with any $g \in \mathcal{S}_{\beta}(\mathbb{R})$ satisfying

$$
\int x^{k} g(x) d x \neq 0, \quad \text { for all } 0 \leq k \leq[\beta], k \in \mathbb{N},
$$

the system, $\left\{\varphi_{i, k}\right\}_{i, k \in \mathbb{Z}} \cup\{g\}$, is complete in $B$. 
It is obvious that $L^{1}(\mathbb{R})$ satisfies $\mathcal{S}_{\beta}(\mathbb{R}) \hookrightarrow L^{1}(\mathbb{R}) \hookrightarrow \mathcal{S}_{\beta}(\mathbb{R})^{*}$ with any $0<\beta<1$ and $\mathcal{S}_{\beta}(\mathbb{R})$ is a dense subset of $L^{1}(\mathbb{R})$. Therefore, condition (5.6) for $g$ becomes

$$
\int g(x) d x \neq 0
$$

Let $\varphi$ be a Daubechies wavelet or a Meyer wavelet and $\Phi$ be its corresponding scaling function, then $\Phi$ satisfies (5.7). Thus, $\left\{\varphi_{i, k}(x)\right\}_{i, k \in \mathbb{Z}} \cup$ $\{\Phi(x)\}$ is a complete system in $L^{1}(\mathbb{R})$. Moreover, using the results derived from Theorem 3.3, we find that, for any fixed $l \in \mathbb{R}$, the linear span of $\left\{\varphi_{i, k}(x)\right\}_{i, k \in \mathbb{Z}} \cup\{\Phi(x-l)\}$ is dense in $L^{1}(\mathbb{R})$.

\subsection{Tail of a generalized MRA}

Let $\alpha, \epsilon>0$ (the $\epsilon$ in Definition 4.1). Given a scaling function, $\Phi \in$ $\mathcal{C}_{\alpha}(\mathbb{R}), \alpha-\epsilon>\eta>0$, for any $j \in \mathbb{Z}$, define the generalized $M R A, T_{j}^{\eta}$, by

$$
T_{j}^{\eta}=\left\{f: f(x)=\sum_{k \in \mathbb{Z}} a_{k} \Phi\left(2^{j} x-k\right) ;\left|a_{k}\right|=O\left(|k|^{\eta}\right)\right\}
$$

The main purpose of this section is to compute the tail of this generalized MRA, that is, $\bigcap_{j \in \mathbb{Z}} T_{j}^{\eta}$. We find that it equals to the set of polynomials of degree at most $[\eta]$. In addition, we show that there is an intimate relation between the annihilator and the Fix-Strang condition. We provide a new proof for the Fix-Strang condition without using Poisson summation formula (see the proof in Theorem 7.4 of [14]). Moreover, we strength the Fix-Strang condition by showing that we have an identity in (1.18). First of all, we define a family of function spaces:

Definition 5.1. For any $\alpha>0$, define $\mathcal{G}_{\alpha}$ to be the function space consisting of those Lebesgue measurable functions $f$ such that

$$
|f(x)| \leq C\left(1+|x|^{\alpha}\right)
$$

for some constant $C>0$ independent of $x \in \mathbb{R}$.

It is easy to see that for any $\alpha, \epsilon>0$, we have $\mathcal{G}_{\alpha} \subset \mathcal{C}_{\alpha}(\mathbb{R})^{*}$. If we define the norm of $\mathcal{G}_{\alpha}$ by $\|f\|_{\mathcal{G}_{\alpha}}=\sup _{x \in \mathbb{R}} \frac{|f(x)|}{1+|x|^{\alpha}}$, then $\mathcal{G}_{\alpha}$ is a Banach space.

Similar to Theorem 3.2, we can prove the following theorem. 
Theorem 5.4. Let $\eta>[\eta] \geq 0$ and $\epsilon>0$. Suppose that $\varphi \in \mathcal{C}_{\alpha}(\mathbb{R})$ where $\alpha>\eta+\epsilon$ is a wavelet for $L^{2}(\mathbb{R})$, then we have

$$
\mathcal{P}_{[\eta]}(\mathbb{R})=\left\{f \in \mathcal{G}_{\eta}:\left\langle f, \varphi_{i, k}\right\rangle=0, \forall i, k \in \mathbb{Z}\right\} .
$$

Proof. By applying identity (5.2), we conclude that for any $f \in \mathcal{G}_{\eta} \subset$ $\mathcal{C}_{\eta}(\mathbb{R})^{*}$ satisfying $\left\langle f, \varphi_{i, k}\right\rangle=0, \forall i, k \in \mathbb{Z}, f$ is equal to a polynomial of degree at most $[\alpha]$. Moreover, we have $\mathcal{P}_{k}(\mathbb{R}) \subset \mathcal{G}_{\eta}$ if and only if $k \leq[\eta]$. Therefore, $f$ is a polynomial of degree at most $[\eta]$. The other direction of the inclusion in (5.10) is guaranteed by (1.8) and $\eta>[\eta]$.

We have the following estimate for $f \in T_{j}^{\eta}$. Without loss of generality, we assume that $j=0$. We find that there exists a constant $C>0$ independent of $x$ such that

$$
\begin{aligned}
|f(x)| & \leq \sum_{k \in \mathbb{Z}}\left|a_{k}\right||\Phi(x-k)| \leq C \sum_{k \in \mathbb{Z}} \frac{|k|^{\eta}}{(1+|x-k|)^{\alpha+1+\epsilon}} \\
& \leq C \int_{\mathbb{R}} \frac{|y|^{\eta}}{(1+|x-y|)^{\alpha+1+\epsilon}} d y \\
& =C \int_{\mathbb{R}} \frac{|x-y|^{\eta}}{(1+|y|)^{\alpha+1+\epsilon}} d y \leq C\left(1+|x|^{\eta}\right) .
\end{aligned}
$$

Therefore, $f \in T_{j}^{\eta}$ implies that $f \in \mathcal{G}_{\eta}$. That is, $T_{j}^{\eta} \subset \mathcal{G}_{\eta}$.

By using the sets, $T_{j}^{\eta}$, we can represent the annihilator for wavelet by the scaling function if the wavelet is generated by a MRA. More specifically, we have

Theorem 5.5. Let $\eta>[\eta] \geq 0$ and $\epsilon>0$. Suppose that $\varphi \in \mathcal{C}_{\alpha}(\mathbb{R})$, where $\alpha>\eta+\epsilon$, is a wavelet generated by a MRA and its corresponding scaling function $\Phi$ belongs to $\mathcal{C}_{\alpha}(\mathbb{R})$. Then,

$$
\left\{f \in \mathcal{G}_{\eta}:\left\langle f, \varphi_{i, k}\right\rangle=0, \forall i, k \in \mathbb{Z}\right\}=\bigcap_{j \in \mathbb{Z}} T_{j}^{\eta} .
$$

Remark 5.1. The result in [8], Chapter 7, Corollary 3.16, states that if $\varphi$ is a wavelet such that $|\hat{\varphi}|$ is continuous and $|\hat{\varphi}(\xi)|=O\left(|\xi|^{-1 / 2-\beta}\right)$ at $\infty$ for some $\beta>0$, then $\varphi$ is a MRA wavelet. Thus, the assumption that the wavelet is a MRA wavelet in Theorem 5.5 is satisfied if $\alpha$ is large enough.

The proof of the above theorem is based on the following convergence theorem for wavelet expansion. 
Theorem 5.6. Let $\alpha>[\alpha] \geq 0$ and $\epsilon>0$. Suppose that the wavelet, $\varphi \in \mathcal{C}_{\alpha}(\mathbb{R})$ is generated by an multiresolution analysis and its scaling function, $\Phi$, belongs to $\mathcal{C}_{\alpha}(\mathbb{R})$, then, for any $f \in \mathcal{C}_{\beta}(\mathbb{R})$ with $\alpha>\beta+\epsilon$ and $l \in \mathbb{Z}$, we have

$$
\lim _{L, L^{\prime \prime} \rightarrow \infty}\left\{\sum_{l \leq i \leq L} \sum_{|k| \leq 2^{L^{\prime \prime}}}\left\langle f, \varphi_{i, k}\right\rangle \varphi_{i, k}+\sum_{|k| \leq 2^{L^{\prime \prime}}}\left\langle f, \Phi_{l-1, k}\right\rangle \Phi_{l-1, k}\right\}=f
$$

in $\mathcal{S}^{\prime}(\mathbb{R})$.

The proof of Theorem 5.6 is similar to the proof of Theorem 2.1, therefore, we leave it to the reader.

Proof of Theorem 5.5. For any $f \in \bigcap_{j \in \mathbb{Z}} T_{j}^{\eta}$ and any fixed $\varphi_{i, m} \in \mathcal{C}_{\eta}(\mathbb{R})$, $i, m \in \mathbb{Z}$, we write $f$ as $f=\sum_{k \in \mathbb{Z}} a_{k} \Phi_{i, k}$, for some $a_{k} \in \mathbb{R}$ satisfying $\left|a_{k}\right|=O\left(|k|^{\eta}\right)$. Since $f \in \bigcap_{j \in \mathbb{Z}} T_{j}^{\eta} \subseteq \mathcal{G}_{\eta}$, it is legitimate to represent $\left\langle f, \varphi_{i, m}\right\rangle$ by $\left\langle f, \varphi_{i, m}\right\rangle=\sum_{k \in \mathbb{Z}} a_{k}\left\langle\Phi_{i, k}, \varphi_{i, m}\right\rangle$. Hence, the result, $\left\langle f, \varphi_{i, m}\right\rangle=0$, for any $i, m \in \mathbb{Z}$, is inherited from $\left\langle\Phi_{i, k}, \varphi_{i, m}\right\rangle=0$, for any $i, k, m \in \mathbb{Z}$.

We now prove the reserve inclusion, $\left\{f \in \mathcal{G}_{\eta}:\left\langle f, \varphi_{i, k}\right\rangle=0, \forall i, k \in \mathbb{Z}\right\} \subseteq$ $\bigcap_{j \in \mathbb{Z}} T_{j}^{\eta}$. For any $f \in \mathcal{G}_{\eta} \subset \mathcal{C}_{\eta}(\mathbb{R})^{*}$, we have a constant, $C>0$, independent of $k$ and $l$ such that

$$
\begin{aligned}
\left|\left\langle f, \Phi_{l, k}\right\rangle\right| & \leq C \int_{\mathbb{R}} \frac{|x|^{\eta}}{\left(1+\left|2^{l} x-k\right|\right)^{\alpha+1+\epsilon}} d x \\
& =C 2^{-l(\eta+1)} \int_{\mathbb{R}} \frac{|y+k|^{\eta}}{(1+|y|)^{\alpha+1+\epsilon}} d y,
\end{aligned}
$$

and, hence, $\left|\left\langle f, \Phi_{l, k}\right\rangle\right| \leq C|k|^{\eta}$.

Thus, for any $l \in \mathbb{Z}$, by Theorem 5.6 , we can write $f$ as

$$
f=\lim _{L^{\prime \prime} \rightarrow \infty} \sum_{|k| \leq 2^{L^{\prime \prime}}}\left\langle f, \Phi_{l, k}\right\rangle \Phi_{l, k}=\sum_{k \in \mathbb{Z}}\left\langle f, \Phi_{l, k}\right\rangle \Phi_{l, k}
$$

with $\left|\left\langle f, \Phi_{l, k}\right\rangle\right|=O\left(|k|^{\eta}\right)$. The existence of the limit on the above identity is asserted by estimate (5.11). Hence, $f \in \bigcap_{j \in \mathbb{Z}} T_{j}^{\eta}$.

Combining (5.10) and (5.12), for any wavelet $\varphi$ satisfying the conditions in Theorem 5.5, we find that

$$
\mathcal{P}_{[\eta]}(\mathbb{R})=\bigcap_{j \in \mathbb{Z}} T_{j}^{\eta} .
$$

Furthermore, the above identity obviously refines the Fix-Strang condition, (1.19), in Theorem 1.2. Finally, if $\varphi, \Phi \in \mathcal{S}(\mathbb{R})$, then, by taking union on both sides of (5.14) with $\eta=k+\frac{1}{2}, k \in \mathbb{N}$, we establish (1.20). 


\subsection{Convergence under inductive limit topology}

This is the only subsection in this article where $\mathcal{S}^{\prime}\left(\mathbb{R}^{n}\right)$ is endowed with a topology different from the weak-star topology.

For any fixed $\epsilon>0$ (the $\epsilon$ in Definition 4.1), we have $\mathcal{S}^{\prime}\left(\mathbb{R}^{n}\right)=$ $\bigcup_{\alpha>0} \mathcal{C}_{\alpha}\left(\mathbb{R}^{n}\right)^{*}$ where the distribution spaces, $\mathcal{C}_{\alpha}\left(\mathbb{R}^{n}\right)^{*}$, with the norm, $\|\cdot\|_{\mathcal{C}_{\alpha}^{*}}=\sup _{\|\psi\|_{\mathcal{C}_{\alpha}}=1}|\langle\cdot, \psi\rangle|$, are Banach spaces; therefore, we can endow $\mathcal{S}^{\prime}\left(\mathbb{R}^{n}\right)$ with the inductive limit topology induced by the inductive system $\left\{\mathcal{C}_{\alpha}\left(\mathbb{R}^{n}\right)^{*}\right\}_{\alpha>0}$ (for the definition of inductive limit topology, see [2], Chapter IV, Section 5). The inductive limit topology is the finest locally convex topology on $\mathcal{S}^{\prime}\left(\mathbb{R}^{n}\right)$ such that all the inclusion mappings, $I_{\alpha}: \mathcal{C}_{\alpha}\left(\mathbb{R}^{n}\right)^{*} \rightarrow \mathcal{S}^{\prime}\left(\mathbb{R}^{n}\right), \alpha>0$, be continuous (in this connection, see [20], p. 515). Thus, by Theorem 5.1, we have

Theorem 5.7. Let $\left\{\varphi_{Q}\right\}_{Q \in \mathcal{Q}} \in \bigcap_{\alpha>0} \mathcal{M}_{\alpha}\left(\mathbb{R}^{n}\right)$ be a frame on $L^{2}\left(\mathbb{R}^{n}\right)$ and $\left\{\psi_{Q}\right\}_{Q \in \mathcal{Q}} \in \bigcap_{\alpha>0} \mathcal{M}_{\alpha}\left(\mathbb{R}^{n}\right)$ be its dual frame. If $f \in \mathcal{S}^{\prime}\left(\mathbb{R}^{n}\right)$ is of order $\omega$, then there exists a sequence of polynomials, $P_{L, L^{\prime}, L^{\prime \prime}}$, of degrees less than or equal to $[\omega]$ such that

$$
\lim _{L, L^{\prime}, L^{\prime \prime} \rightarrow \infty}\left\{\sum_{i=-L^{\prime}}^{L} \sum_{|k| \leq \delta(i) 2^{L^{\prime \prime}}}\left\langle f, \psi_{Q_{i, k}}\right\rangle \varphi_{Q_{i, k}}-P_{L, L^{\prime}, L^{\prime \prime}}\right\}=f
$$

in $\mathcal{S}^{\prime}\left(\mathbb{R}^{n}\right)$ under the inductive limit topology. If $\left\{\varphi_{Q}\right\}_{Q \in \mathcal{Q}},\left\{\psi_{Q}\right\}_{Q \in \mathcal{Q}} \in$ $\mathcal{M}_{\alpha}\left(\mathbb{R}^{n}\right)$, then, for any $f \in \mathcal{C}_{\beta}\left(\mathbb{R}^{n}\right)^{*}$ with $\alpha>\beta+\epsilon$, the limit, (5.2), converges in $\mathcal{S}^{\prime}\left(\mathbb{R}^{n}\right)$ under the inductive limit topology.

\section{§6. Reproducing formula in $\mathcal{S}^{\prime}\left(\mathbb{R}^{n}\right) / \mathcal{P}\left(\mathbb{R}^{n}\right)$}

With respect to our definition of frame, the functions, $\varphi$ and $\psi$, used to define the $\varphi-\psi$ transform in [6] are not necessarily a frame and its corresponding dual frame, respectively. On the other hand, the convergence of the $\varphi-\psi$ transform is included in the following generalization of Theorem 2.1. The following theorem states that a reproducing formula for functions in $L^{p}\left(\mathbb{R}^{n}\right),(6.1)$, automatically produces a reproducing formula for distributions in $\mathcal{S}^{\prime}\left(\mathbb{R}^{n}\right) / \mathcal{P}\left(\mathbb{R}^{n}\right),(6.2)$, when the analyzing family and the reconstructing family, $\left\{\varphi_{Q}\right\}_{Q \in \mathcal{Q}}$ and $\left\{\psi_{Q}\right\}_{Q \in \mathcal{Q}}$, satisfy a mild assumption, $\left\{\varphi_{Q}\right\}_{Q \in \mathcal{Q}},\left\{\psi_{Q}\right\}_{Q \in \mathcal{Q}} \in \bigcap_{\alpha} \mathcal{M}_{\alpha}\left(\mathbb{R}^{n}\right)$. 
Theorem 6.1. Let $1<p<\infty$. Suppose that $\left\{\varphi_{Q}\right\}_{Q \in \mathcal{Q}},\left\{\psi_{Q}\right\}_{Q \in \mathcal{Q}} \in$ $\bigcap_{\alpha} \mathcal{M}_{\alpha}\left(\mathbb{R}^{n}\right)$ satisfy, for any $f \in L^{p}\left(\mathbb{R}^{n}\right)$,

$$
f=\sum_{Q \in \mathcal{Q}}\left\langle f, \varphi_{Q}\right\rangle \psi_{Q}
$$

in $L^{p}\left(\mathbb{R}^{n}\right)$. Then, for any $f \in \mathcal{S}^{\prime}\left(\mathbb{R}^{n}\right) / \mathcal{P}\left(\mathbb{R}^{n}\right)$,

$$
f=\sum_{Q \in \mathcal{Q}}\left\langle f, \psi_{Q}\right\rangle \varphi_{Q}=\sum_{Q \in \mathcal{Q}}\left\langle f, \varphi_{Q}\right\rangle \psi_{Q}
$$

in $\mathcal{S}^{\prime}\left(\mathbb{R}^{n}\right) / \mathcal{P}\left(\mathbb{R}^{n}\right)$.

For $\alpha>\beta+\epsilon$ (the $\epsilon$ in Definition 2.1) and $[\alpha]=[\beta]$, suppose that $\left\{\varphi_{Q}\right\}_{Q \in \mathcal{Q}}$ and $\left\{\psi_{Q}\right\}_{Q \in \mathcal{Q}}$ belong to $\mathcal{M}_{\alpha}\left(\mathbb{R}^{n}\right)$ and satisfy (6.1). Then, for any $f \in \mathcal{C}_{\beta}\left(\mathbb{R}^{n}\right)^{*} / \mathcal{P}_{[\alpha]}\left(\mathbb{R}^{n}\right)$, where $\mathcal{P}_{[\alpha]}\left(\mathbb{R}^{n}\right)$ denotes the space of polynomials of degrees at most $[\alpha]$, we have

$$
f=\sum_{Q \in \mathcal{Q}}\left\langle f, \psi_{Q}\right\rangle \varphi_{Q}=\sum_{Q \in \mathcal{Q}}\left\langle f, \varphi_{Q}\right\rangle \psi_{Q}
$$

in $\mathcal{S}^{\prime}\left(\mathbb{R}^{n}\right) / \mathcal{P}_{[\alpha]}\left(\mathbb{R}^{n}\right)$.

We omit the proof for Theorem 6.1. In fact, the proof for the first identity in (6.2) is the same as the proofs for Theorem 2.1 and Theorem 4.1. We prove Theorem 2.1 by duality, therefore, in the first identity of (6.2), we have to interchange the roles of $\varphi$ and $\psi$. On the other hand, by (6.1), for any fixed $g \in L^{p^{\prime}}\left(\mathbb{R}^{n}\right)$, where $p^{\prime}$ is the conjugate of $p$, we have

$$
\langle g, f\rangle=\sum_{Q \in \mathcal{Q}}\left\langle g, \psi_{Q}\right\rangle\left\langle\varphi_{Q}, f\right\rangle, \quad \forall f \in L^{p}\left(\mathbb{R}^{n}\right)
$$

(recall that we endow the pairing, $\langle\cdot, \cdot\rangle$, with the antilinear structure). Thus, we assert that

$$
g=\sum_{Q \in \mathcal{Q}}\left\langle g, \psi_{Q}\right\rangle \varphi_{Q}, \quad \forall g \in L^{p^{\prime}}\left(\mathbb{R}^{n}\right)
$$

and, hence, the second identity in (6.2) holds by using (6.3).

Notice that, for all $f \in \mathcal{S}^{\prime}\left(\mathbb{R}^{n}\right) / \mathcal{P}\left(\mathbb{R}^{n}\right)$, the pairings, $\left\langle f, \psi_{Q}\right\rangle$ and $\left\langle f, \varphi_{Q}\right\rangle$, are well defined if and only if $\left\{\psi_{Q}\right\}_{Q \in \mathcal{Q}}$ and $\left\{\varphi_{Q}\right\}_{Q \in \mathcal{Q}}$ are subsets of $\mathcal{S}_{0}\left(\mathbb{R}^{n}\right)$. 
Therefore, the assumption, $\left\{\varphi_{Q}\right\}_{Q \in \mathcal{Q}},\left\{\psi_{Q}\right\}_{Q \in \mathcal{Q}} \in \bigcap_{\alpha} \mathcal{M}_{\alpha}\left(\mathbb{R}^{n}\right)$, is indispensable if the analyzing family and the reconstructing family are "welllocalized" with respect to the set of dyadic cubes, $\mathcal{Q}$. The author has no idea on whether we can relax the condition to $\left\{\varphi_{Q}\right\}_{Q \in \mathcal{Q}},\left\{\psi_{Q}\right\}_{Q \in \mathcal{Q}} \subset \mathcal{S}_{0}\left(\mathbb{R}^{n}\right)$.

Observe that the roles of the analyzing family and the reconstructing family in (6.2) can be interchanged. In fact, this is a remarkable feature of reproducing formula in $\mathcal{S}^{\prime}\left(\mathbb{R}^{n}\right) / \mathcal{P}\left(\mathbb{R}^{n}\right)$. The following theorem shows that the notions of analyzing family and reconstructing family are indistinguishable for reproducing formula in $\mathcal{S}^{\prime}\left(\mathbb{R}^{n}\right) / \mathcal{P}\left(\mathbb{R}^{n}\right)$.

Theorem 6.2. Let $\left\{\varphi_{Q}\right\}_{Q \in \mathcal{Q}},\left\{\psi_{Q}\right\}_{Q \in \mathcal{Q}} \in \bigcap_{\alpha} \mathcal{M}_{\alpha}\left(\mathbb{R}^{n}\right)$ satisfy,

$$
f=\sum_{Q \in \mathcal{Q}}\left\langle f, \psi_{Q}\right\rangle \varphi_{Q}, \quad \forall f \in \mathcal{S}^{\prime}\left(\mathbb{R}^{n}\right) / \mathcal{P}\left(\mathbb{R}^{n}\right),
$$

in $\mathcal{S}^{\prime}\left(\mathbb{R}^{n}\right) / \mathcal{P}\left(\mathbb{R}^{n}\right)$. Then, we have

$$
f=\sum_{Q \in \mathcal{Q}}\left\langle f, \varphi_{Q}\right\rangle \psi_{Q}, \quad \forall f \in \mathcal{S}^{\prime}\left(\mathbb{R}^{n}\right) / \mathcal{P}\left(\mathbb{R}^{n}\right),
$$

in $\mathcal{S}^{\prime}\left(\mathbb{R}^{n}\right) / \mathcal{P}\left(\mathbb{R}^{n}\right)$

Proof. Applying (6.4) to any $g \in \mathcal{S}_{0}\left(\mathbb{R}^{n}\right) \subset \mathcal{S}^{\prime}\left(\mathbb{R}^{n}\right) / \mathcal{P}\left(\mathbb{R}^{n}\right)$, we obtain

$$
g=\sum_{Q \in \mathcal{Q}}\left\langle g, \psi_{Q}\right\rangle \varphi_{Q}, \quad \forall g \in \mathcal{S}_{0}\left(\mathbb{R}^{n}\right)
$$

in $\mathcal{S}^{\prime}\left(\mathbb{R}^{n}\right) / \mathcal{P}\left(\mathbb{R}^{n}\right)$. Using the idea for proving Theorem 4.1, we find that $\sum_{Q \in \mathcal{Q}}\left\langle g, \psi_{Q}\right\rangle \varphi_{Q}$ converges in the topology of $\mathcal{S}_{0}\left(\mathbb{R}^{n}\right)$. By $(6.6)$, the limit of $\sum_{Q \in \mathcal{Q}}\left\langle g, \psi_{Q}\right\rangle \varphi_{Q}$ in $\mathcal{S}_{0}\left(\mathbb{R}^{n}\right)$ must be equal to $g$, hence, we have a stronger result,

$$
g=\sum_{Q \in \mathcal{Q}}\left\langle g, \psi_{Q}\right\rangle \varphi_{Q}, \quad \forall g \in \mathcal{S}_{0}\left(\mathbb{R}^{n}\right)
$$

in $\mathcal{S}_{0}\left(\mathbb{R}^{n}\right)$. For any $g \in \mathcal{S}_{0}\left(\mathbb{R}^{n}\right)$, it is legitimate to apply $f \in \mathcal{S}^{\prime}\left(\mathbb{R}^{n}\right) / \mathcal{P}\left(\mathbb{R}^{n}\right)$ on both sides of $(6.7)$ because $\mathcal{S}^{\prime}\left(\mathbb{R}^{n}\right) / \mathcal{P}\left(\mathbb{R}^{n}\right)$ is the dual space of $\mathcal{S}_{0}\left(\mathbb{R}^{n}\right)$. We assert that

$$
\langle f, g\rangle=\sum_{Q \in \mathcal{Q}}\left\langle f, \varphi_{Q}\right\rangle\left\langle\psi_{Q}, g\right\rangle, \quad \forall g \in \mathcal{S}_{0}\left(\mathbb{R}^{n}\right) .
$$


Thus, $\sum_{Q \in \mathcal{Q}}\left\langle f, \varphi_{Q}\right\rangle \psi_{Q}$ is well defined and belongs to the dual of $\mathcal{S}_{0}\left(\mathbb{R}^{n}\right)$. By (6.8), we prove that

$$
f=\sum_{Q \in \mathcal{Q}}\left\langle f, \varphi_{Q}\right\rangle \psi_{Q}, \quad \forall f \in \mathcal{S}^{\prime}\left(\mathbb{R}^{n}\right) / \mathcal{P}\left(\mathbb{R}^{n}\right),
$$

in $\mathcal{S}^{\prime}\left(\mathbb{R}^{n}\right) / \mathcal{P}\left(\mathbb{R}^{n}\right)$.

\section{§7. Proof of Theorem 4.1}

\subsection{Technical results}

We need some technical results for the proof of Theorem 4.1. The following lemma provides an important estimate on $\left|\left\langle g, \varphi_{Q}\right\rangle\right|$ where $\left\{\varphi_{Q}\right\}_{Q} \in$ $\mathcal{M}_{\alpha}\left(\mathbb{R}^{n}\right)$ and $g \in \mathcal{C}_{\alpha}\left(\mathbb{R}^{n}\right)$.

Lemma 7.1. Let $\alpha>[\alpha] \geq 0$ and $\left\{\varphi_{Q}\right\}_{Q} \in \mathcal{M}_{\alpha}\left(\mathbb{R}^{n}\right)$. If $g \in \mathcal{C}_{\alpha}\left(\mathbb{R}^{n}\right)$ satisfies the vanishing moments,

$$
\int_{\mathbb{R}^{n}} x^{\lambda} g(x) d x=0 \quad \text { for } \lambda \in \mathbb{N}^{n} \text { and } 0 \leq|\lambda| \leq[\alpha],
$$

then there exists a constant $C>0$, independent of $Q=Q_{i, k} \in \mathcal{Q}$, such that

$$
\left|\left\langle g, \varphi_{Q_{i, k}}\right\rangle\right| \leq C\|g\|_{\mathcal{C}_{\alpha}}\left(\min \left(2^{i}, 2^{-i}\right)\right)^{1 / 2+\alpha}\left(1+\frac{\left|2^{-i} k\right|}{\max \left(1,2^{-i}\right)}\right)^{-\alpha-n-\epsilon} .
$$

Proof. If $2^{-i} \geq 1$, by applying Lemma B.1 of [6] with $R=S=\alpha+n+\epsilon$, $L=[\alpha], \theta=\alpha-[\alpha], x=2^{-i} k$ and $x_{1}=0\left(R, S, L, \theta, x\right.$ and $x_{1}$ are the notations in Lemma B.1 of [6]), we have a constant $C>0$ independent of $i, k$ and $g$ such that

$$
\left|\left\langle g, \varphi_{Q_{i, k}}\right\rangle\right| \leq C\|g\|_{\mathcal{C}_{\alpha}} 2^{i(n / 2+\alpha)}(1+|k|)^{-\alpha-n-\epsilon} .
$$

As $\left\{\varphi_{Q}\right\}_{Q} \in \mathcal{M}_{\alpha}\left(\mathbb{R}^{n}\right)$, satisfies the vanishing moment condition, (2.1). Therefore, interchanging the role of $\varphi_{Q_{i, k}}$ and $g$, if $2^{-i}<1$, we then have

$$
\left|\left\langle g, \varphi_{Q_{i, k}}\right\rangle\right| \leq C\|g\|_{\mathcal{C}_{\alpha}} 2^{-i(n / 2+\alpha)}\left(1+\left|2^{-i} k\right|\right)^{-\alpha-n-\epsilon},
$$

which is our desired result.

Remark 7.1. We impose the assumption, $\alpha>[\alpha]$, in Lemma 7.1 and Theorem 4.1 in order to fulfill the condition, $\alpha-[\alpha]=\theta>0$, given by Lemma B.1 of [6]. 
Observe that the relatively large frame coefficients, $\left|\left\langle g, \varphi_{Q_{i, k}}\right\rangle\right|$, for any fixed $i=i_{0} \geq 0$ and $i=i_{1}<0$, are located on the sets, $\left\{\left(i_{0}, k\right):|k| \leq\right.$ $\left.2^{i+L^{\prime \prime}}\right\}$ and $\left\{\left(i_{1}, k\right):|k| \leq 2^{L^{\prime \prime}}\right\}$, respectively. Therefore, the truncation set, $T\left(L, L^{\prime}, L^{\prime \prime}\right)$, is more well-adapted to our setting than $\tilde{T}\left(L, L^{\prime}, L^{\prime \prime}\right)$ and the above estimate suggests the use of $\delta(i)$.

We need one more result before we present the proof of Theorem 4.1. The following lemma, Lemma 7.2, is a discrete version of Lemma B.2 in [6].

Lemma 7.2. If $i \in \mathbb{Z}^{n}, 1 \geq 2^{-i}, \epsilon>0$ and $\alpha>\beta>0$, then we have the following results.

(1) We have a constant $C>0$ such that, for any $i>0$ and $x \in \mathbb{R}^{n}$,

$$
\begin{aligned}
& \sum_{k \in \mathbb{Z}^{n}}\left(1+\left|2^{-i} k\right|\right)^{-\alpha-n-\epsilon}\left(1+2^{i}\left|x-2^{-i} k\right|\right)^{-\alpha-n-\epsilon} \\
& \quad \leq C(1+|x|)^{-\alpha-n-\epsilon} .
\end{aligned}
$$

(2) We have a constant $C>0$ such that, for any $i>0, x \in \mathbb{R}^{n}$ and $M>2$,

$$
\begin{aligned}
& \sum_{\substack{k \in \mathbb{Z}^{n} \\
\left|2^{-i} k\right| \geq M}}\left(1+\left|2^{-i} k\right|\right)^{-\alpha-n-\epsilon}\left(1+2^{i}\left|x-2^{-i} k\right|\right)^{-\alpha-n-\epsilon} \\
& \leq C M^{\beta-\alpha}(1+|x|)^{-\beta-n-\epsilon} .
\end{aligned}
$$

Proof. Since $1 \geq 2^{-i}$, there exists a constant $C>0$ such that, for any $y \in 2^{-i}\left([0,1]^{n}+k\right)$,

$$
C^{-1}(1+|y|)^{-\alpha-n-\epsilon} \leq\left(1+\left|2^{-i} k\right|\right)^{-\alpha-n-\epsilon} \leq C(1+|y|)^{-\alpha-n-\epsilon} .
$$

Similarly, for any $y \in 2^{-i}\left([0,1]^{n}+k\right)$ and $x \in \mathbb{R}^{n}$, we find that $C^{-1}\left(1+2^{i}|x-y|\right)^{-\alpha-n-\epsilon} \leq\left(1+2^{i}\left|x-2^{-i} k\right|\right)^{-\alpha-n-\epsilon} \leq C\left(1+2^{i}|x-y|\right)^{-\alpha-n-\epsilon}$. Therefore, by Lemma B.2 of [6], we have

$$
\begin{aligned}
& \sum_{k \in \mathbb{Z}^{n}}\left(1+\left|2^{-i} k\right|\right)^{-\alpha-n-\epsilon}\left(1+2^{i}\left|x-2^{-i} k\right|\right)^{-\alpha-n-\epsilon} \\
& \leq C \sum_{k \in \mathbb{Z}^{n}} 2^{i} \int_{2^{-i}\left([0,1]^{n}+k\right)}(1+|y|)^{-\alpha-n-\epsilon}\left(1+2^{i}|x-y|\right)^{-\alpha-n-\epsilon} d y \\
& \leq C 2^{i} \int_{\mathbb{R}^{n}}(1+|y|)^{-\alpha-n-\epsilon}\left(1+2^{i}|x-y|\right)^{-\alpha-n-\epsilon} d y \\
& \leq C(1+|x|)^{-\alpha-n-\epsilon} .
\end{aligned}
$$


The second part is similar to the first part except that we take integration over the domain, $|y|>M-1$, instead of $\mathbb{R}^{n}$. Since $\alpha>\beta$, we obtain

$$
\begin{aligned}
& \sum_{\substack{k \in \mathbb{Z}^{n} \\
\left|2^{-i} k\right| \geq M}}\left(1+\left|2^{-i} k\right|\right)^{-\alpha-n-\epsilon}\left(1+2^{i}\left|x-2^{-i} k\right|\right)^{-\alpha-n-\epsilon} \\
& \leq C \sum_{\substack{k \in \mathbb{Z}^{n} \\
\left|2^{-i} k\right| \geq M}} 2^{i} \int_{2^{-i}\left([0,1]^{n}+k\right)}(1+|y|)^{-\alpha-n-\epsilon}\left(1+2^{i}|x-y|\right)^{-\alpha-n-\epsilon} d y \\
& \leq C 2^{i} M^{\beta-\alpha} \int_{|y| \geq M-1}(1+|y|)^{-\beta-n-\epsilon}\left(1+2^{i}|x-y|\right)^{-\beta-n-\epsilon} d y \\
& \leq C M^{\beta-\alpha}(1+|x|)^{-\beta-n-\epsilon} .
\end{aligned}
$$

\subsection{The proof}

We are ready to prove Theorem 4.1. The proof is based on the ideas in Lemma 9.14 of [6], Theorem 3.2 and Theorem 3.3 in Chapter 3 of [16], Theorem 3 in the Appendix of [7] and, especially, Proposition 1.7 of [11]. However, we cannot directly apply the above results to conclude our theorem. For instance, as mentioned in [6] (see p. 107 of [6]), the proof of Lemma 9.14 in [6] cannot be generalized to estimate the derivatives of the "molecules decomposition" and, hence, cannot be used to estimate the derivatives of the truncated frame expansion, (4.6).

We prove Theorem 4.1 by a standard technique of splitting the frame expansion into two components (see Lemma 9.14 of [6]). The first component contains those terms, $\left\langle g, \varphi_{Q_{i, k}}\right\rangle, Q_{i, k}=2^{-i}\left([0,1]^{n}+k\right)$, when $\left|Q_{i, k}\right|$ is small and the second component is for those terms, when $\left|Q_{i, k}\right|$ is large (the terms, $I^{\prime}$ and $I I^{\prime}$, in (7.3), respectively). Under our situation, we need a modification adapted to our truncation on the frame expansion. For each component, we insert an extra series including those $\left\langle g, \varphi_{Q_{i, k}}\right\rangle$ with $Q_{i, k}$ "having a long distance" from the origin in order to compensate for the truncation on $k$ (the terms, $I^{\prime \prime}$ and $I I^{\prime \prime}$, in (7.3)).

We are going to prove that

$$
\sum_{i=-L^{\prime}}^{L} \sum_{|k| \leq \delta(i) 2^{L^{\prime \prime}}}\left\langle g, \varphi_{Q_{i, k}}\right\rangle \psi_{Q_{i, k}},
$$

$L, L^{\prime}, L^{\prime \prime} \in \mathbb{N}$ is a Cauchy sequence and converges to $g$ in $\mathcal{C}_{\beta}\left(\mathbb{R}^{n}\right)$. Let $L$, $L^{\prime}, L^{\prime \prime}, N, N^{\prime}$ and $N^{\prime \prime}$ be positive integers. Without loss of generality, we 
assume that $\|g\|_{\mathcal{C}_{\alpha}}=1, N>L, N^{\prime}>L^{\prime}$ and $N^{\prime \prime}>L^{\prime \prime}$. For $\gamma \in \mathbb{N}^{n}$ with $0 \leq|\gamma| \leq[\beta]$, we write

$$
\begin{aligned}
\mid \partial^{\gamma}\left(\sum_{i=-N^{\prime}}^{N} \sum_{|k| \leq \delta(i) 2^{N^{\prime \prime}}}\left\langle g, \varphi_{Q_{i, k}}\right\rangle \psi_{Q_{i, k}}(x)\right. \\
\left.\quad-\sum_{i=-L^{\prime}}^{L} \sum_{|k| \leq \delta(i) 2^{L^{\prime \prime}}}\left\langle g, \varphi_{Q_{i, k}}\right\rangle \psi_{Q_{i, k}}(x)\right) \mid \\
\leq \sum_{i=L+1}^{\infty} \sum_{k \in \mathbb{Z}^{n}}\left|\left\langle g, \varphi_{Q_{i, k}}\right\rangle\right|\left|\partial^{\gamma} \psi_{Q_{i, k}}(x)\right| \\
\quad+\sum_{i=-\infty} \sum_{k \in \mathbb{Z}^{n}}\left|\left\langle g, \varphi_{Q_{i, k}}\right\rangle\right|\left|\partial^{\gamma} \psi_{Q_{i, k}}(x)\right| \\
\quad+\sum_{i=0}^{L} \sum_{|k|>2^{i+L^{\prime \prime}}}\left|\left\langle g, \varphi_{Q_{i, k}}\right\rangle\right|\left|\partial^{\gamma} \psi_{Q_{i, k}}(x)\right| \\
\quad+\sum_{i=-L^{\prime}}^{-1} \sum_{|k|>2^{L^{\prime \prime}}}\left|\left\langle g, \varphi_{Q_{i, k}}\right\rangle\right|\left|\partial^{\gamma} \psi_{Q_{i, k}}(x)\right| \\
=I^{\prime}+I I^{\prime}+I^{\prime \prime}+I I^{\prime \prime},
\end{aligned}
$$

according to the definition of $\delta(i)$.

For $I^{\prime}$, by Lemma 7.1, we have a constant $C>0$ such that

$$
\begin{gathered}
I^{\prime} \leq C \sum_{i=L+1}^{\infty} \sum_{k \in \mathbb{Z}^{n}}\left(1+\left|2^{-i} k\right|\right)^{-\alpha-n-\epsilon} 2^{-i \alpha-i n / 2} 2^{i n / 2+|\gamma| i} \\
\times\left(1+2^{i}\left|x-2^{-i} k\right|\right)^{-\alpha-n-\epsilon} \\
\leq C \sum_{i=L+1}^{\infty} 2^{-i(\alpha-|\gamma|)} \sum_{k \in \mathbb{Z}^{n}}\left(1+\left|2^{-i} k\right|\right)^{-\alpha-n-\epsilon} \\
\times\left(1+2^{i}\left|x-2^{-i} k\right|\right)^{-\alpha-n-\epsilon} .
\end{gathered}
$$

Furthermore, we find that

$$
I^{\prime} \leq C 2^{(-L-1)(\alpha-|\gamma|)}(1+|x|)^{-\beta-n-\epsilon} \leq C 2^{(-L-1)(\alpha-\beta)}(1+|x|)^{-\beta-n-\epsilon}
$$

by the first part of Lemma 7.2 and $\alpha>\beta \geq|\gamma|$. 
For $I I^{\prime}$, we have

$$
\begin{aligned}
I I^{\prime} \leq & C \sum_{i=-\infty}^{-L^{\prime}-1} \sum_{k \in \mathbb{Z}^{n}}(1+|k|)^{-\alpha-n-\epsilon} 2^{i(\alpha+n / 2)} 2^{i n / 2+|\gamma| i} \\
& \times\left(1+2^{i}\left|x-2^{-i} k\right|\right)^{-\alpha-n-\epsilon} \\
\leq & C \sum_{i=-\infty}^{-L^{\prime}-1} 2^{i(n+|\gamma|+\alpha)}\left(\frac{1}{1+2^{i}|x|}\right)^{\beta+n+\epsilon} \\
& \leq C \sum_{i=-\infty}^{-L^{\prime}-1} 2^{i(n+|\gamma|+\alpha)}\left(\frac{2^{-i}}{2^{-i}+|x|}\right)^{\beta+n+\epsilon}
\end{aligned}
$$

and the second inequality holds because

$$
\sum_{k \in \mathbb{Z}^{n}}(1+|k|)^{-\alpha-n-\epsilon}\left(1+2^{i}\left|x-2^{-i} k\right|\right)^{-\alpha-n-\epsilon} \leq C\left(1+2^{i}|x|\right)^{-\alpha-n-\epsilon}
$$

for some constant $C>0$ independent of $i$ and $x$ (for the proof of (7.4), see [1], p. 67), and $\alpha>\beta+\epsilon>\beta$.

By using the fact that $i<0$ and $\alpha>\beta+\epsilon$, we obtain

$$
\begin{aligned}
I I^{\prime} & \leq C\left(\sum_{i=-\infty}^{-L^{\prime}-1} 2^{i(n+|\gamma|+\alpha-\beta-n-\epsilon)}\right)\left(\frac{1}{1+|x|}\right)^{\beta+n+\epsilon} \\
& \leq C 2^{\left(-L^{\prime}-1\right)(\alpha-\beta-\epsilon)}\left(\frac{1}{1+|x|}\right)^{\beta+n+\epsilon} .
\end{aligned}
$$

The estimates for $I^{\prime \prime}$ and $I I^{\prime \prime}$ are similar to the estimates for $I^{\prime}$ and $I I^{\prime}$ except that, for $I^{\prime \prime}$, we use the second part of Lemma 7.2 with $M=2^{L^{\prime \prime}}$ instead of the first part. We have

$$
I^{\prime \prime}+I I^{\prime \prime} \leq C 2^{-L^{\prime \prime}(\alpha-\beta)}(1+|x|)^{-\beta-n-\epsilon} .
$$

Notice that the term, $\delta(i)$, plays a crucial role in the estimate for $I I^{\prime \prime}$ when $\gamma=0$. This will be further justified by the convergence of the truncated frame expansions by using truncation sets different from $T\left(L, L^{\prime}, L^{\prime \prime}\right)$, see Section 8.1.

By combining the estimates for $I^{\prime}, I I^{\prime}, I^{\prime \prime}$ and $I I^{\prime \prime}$, we establish the 
inequality (recall (4.1) for the definition of $\|\cdot\|_{\beta *}$ ),

$$
\begin{aligned}
& \left\|\sum_{i=-N^{\prime}}^{N} \sum_{|k| \leq \delta(i) 2^{N^{\prime \prime}}}\left\langle g, \varphi_{Q_{i, k}}\right\rangle \psi_{Q_{i, k}}-\sum_{i=-L^{\prime}}^{L} \sum_{|k| \leq \delta(i) 2^{L^{\prime \prime}}}\left\langle g, \varphi_{Q_{i, k}}\right\rangle \psi_{Q_{i, k}}\right\|_{\beta *} \\
& \quad \leq C\left(2^{(-L-1)(\alpha-\beta)}+2^{\left(-L^{\prime}-1\right)(\alpha-\beta-\epsilon)}+2^{-L^{\prime \prime}(\alpha-\beta)}\right) .
\end{aligned}
$$

For any fixed $N>L>0, N^{\prime}>L^{\prime}>0$ and $N^{\prime \prime}>L^{\prime \prime}>0$, let

$\Psi(x)=\sum_{i=-N^{\prime}}^{N} \sum_{|k| \leq \delta(i) 2^{N^{\prime \prime}}}\left\langle g, \varphi_{Q_{i, k}}\right\rangle \psi_{Q_{i, k}}(x)-\sum_{i=-L^{\prime}}^{L} \sum_{|k| \leq \delta(i) 2^{L^{\prime \prime}}}\left\langle g, \varphi_{Q_{i, k}}\right\rangle \psi_{Q_{i, k}}(x)$

and

$$
\omega\left(L, L^{\prime}, L^{\prime \prime}\right)=2^{(-L-1)(\alpha-\beta)}+2^{\left(-L^{\prime}-1\right)(\alpha-\beta-\epsilon)}+2^{-L^{\prime \prime}(\alpha-\beta)} .
$$

For the estimate of (recall (4.2) for the definition of $\|\cdot\|_{\beta * *}$ )

$$
\left\|\sum_{i=-N^{\prime}}^{N} \sum_{|k| \leq \delta(i) 2^{N^{\prime \prime}}}\left\langle g, \varphi_{Q_{i, k}}\right\rangle \psi_{Q_{i, k}}-\sum_{i=-L^{\prime}}^{L} \sum_{|k| \leq \delta(i) L^{L^{\prime \prime}}}\left\langle g, \varphi_{Q_{i, k}}\right\rangle \psi_{Q_{i, k}}\right\|_{\beta * *},
$$

we assume that $|x-y| \leq 1$. Otherwise, we have $1<|x-y|$, and, hence, for $\gamma \in \mathbb{N}^{n}$ and $|\gamma|=[\beta]$, we obtain

$$
\begin{aligned}
\left|\partial^{\gamma} \Psi(x)-\partial^{\gamma} \Psi(y)\right| & \leq\left|\partial^{\gamma} \Psi(x)\right|+\left|\partial^{\gamma} \Psi(y)\right| \\
& \leq C \omega\left(L, L^{\prime}, L^{\prime \prime}\right)\left((1+|x|)^{-\beta-n-\epsilon}+(1+|y|)^{-\beta-n-\epsilon}\right) \\
& \leq C \omega\left(L, L^{\prime}, L^{\prime \prime}\right)|x-y|^{\beta-[\beta]} \sup _{|z| \leq|x-y|}(1+|x-z|)^{-\beta-n-\epsilon}
\end{aligned}
$$

by the estimates of $I^{\prime}, I I^{\prime}, I^{\prime \prime}$ and $I I^{\prime \prime}$.

Let $l \in \mathbb{N}$ satisfy $2^{-l} \leq|x-y|<2^{-l+1}$. For $\gamma \in \mathbb{N}^{n}$ and $|\gamma|=[\beta]$, we have

(7.8) $\left|\partial^{\gamma} \Psi(x)-\partial^{\gamma} \Psi(y)\right|$

$$
\begin{aligned}
\leq & \sum_{i=\max (L+1, l)}^{\infty} \sum_{k \in \mathbb{Z}^{n}}\left|\left\langle g, \varphi_{Q_{i, k}}\right\rangle\right|\left(\left|\partial^{\gamma} \psi_{Q_{i, k}}(x)\right|+\left|\partial^{\gamma} \psi_{Q_{i, k}}(y)\right|\right) \\
& +\sum_{i=L+1}^{\max (L+1, l)-1} \sum_{k \in \mathbb{Z}^{n}}\left|\left\langle g, \varphi_{Q_{i, k}}\right\rangle\right|\left|\partial^{\gamma} \psi_{Q_{i, k}}(x)-\partial^{\gamma} \psi_{Q_{i, k}}(y)\right|
\end{aligned}
$$




$$
\begin{aligned}
& +\sum_{i=-\infty}^{-L^{\prime}-1} \sum_{k \in \mathbb{Z}^{n}}\left|\left\langle g, \varphi_{Q_{i, k}}\right\rangle\right|\left|\partial^{\gamma} \psi_{Q_{i, k}}(x)-\partial^{\gamma} \psi_{Q_{i, k}}(y)\right| \\
& +\sum_{i=\min (l, L)+1}^{L} \sum_{|k|>2^{L^{\prime \prime}+i}}\left|\left\langle g, \varphi_{Q_{i, k}}\right\rangle\right|\left(\left|\partial^{\gamma} \psi_{Q_{i, k}}(x)\right|+\left|\partial^{\gamma} \psi_{Q_{i, k}}(y)\right|\right) \\
& +\sum_{i=0}^{\min (l, L)} \sum_{|k|>2^{L^{\prime \prime}+i}}\left|\left\langle g, \varphi_{Q_{i, k}}\right\rangle\right|\left|\partial^{\gamma} \psi_{Q_{i, k}}(x)-\partial^{\gamma} \psi_{Q_{i, k}}(y)\right| \\
& +\sum_{i=-L^{\prime}}^{-1} \sum_{|k|>2^{L^{\prime \prime}}}\left|\left\langle g, \varphi_{Q_{i, k}}\right\rangle\right|\left|\partial^{\gamma} \psi_{Q_{i, k}}(x)-\partial^{\gamma} \psi_{Q_{i, k}}(y)\right| \\
& =I I I^{\prime}+I V^{\prime}+V^{\prime}+I I I^{\prime \prime}+I V^{\prime \prime}+V^{\prime \prime} .
\end{aligned}
$$

The summation, $I I I^{\prime}$, takes over those terms, $\left|\left\langle g, \varphi_{Q_{i, k}}\right\rangle\right|$, such that the length of $Q_{i, k}, l\left(Q_{i, k}\right)=2^{-i}$, satisfies $l\left(Q_{i, k}\right) \leq \min \left(|x-y|, 2^{-L-1}\right)$. The summation for $I V^{\prime}$ includes the terms, $\left|\left\langle g, \varphi_{Q_{i, k}}\right\rangle\right|$, with $|x-y| \leq l\left(Q_{i, k}\right) \leq$ $2^{-L-1}$. If $L+1 \geq l$, then $I V^{\prime}$ vanishes. Thus, we assume $l>i$ on estimating $I V^{\prime}$. The term, $V^{\prime}$, collects the remaining. That is, those $\left|\left\langle g, \varphi_{Q_{i, k}}\right\rangle\right|$ with $Q_{i, k}$ satisfy $2^{L^{\prime}+1} \leq l\left(Q_{i, k}\right)$.

The terms, $I I I^{\prime \prime}, I V^{\prime \prime}$ and $V^{\prime \prime}$, are similar to the terms, $I I I^{\prime}, I V^{\prime}$ and $V^{\prime}$, respectively, except that they take over the summation for $k$ on the region, $|k|>\delta(i) 2^{L^{\prime \prime}}$. Moreover, we do not have $I I I^{\prime \prime}$ if $L \leq l$ and, hence, $i>l$ when we deal with $I I I^{\prime \prime}$.

We first consider $I I I^{\prime}$. Under the range for $i$ on $I I I^{\prime}, 2^{-i} \leq 2^{-l} \leq$ $|x-y| \leq 1$, we find that

$$
\begin{aligned}
I I I^{\prime}= & \sum_{i=\max (L+1, l)}^{\infty} \sum_{k \in \mathbb{Z}^{n}}\left|\left\langle g, \varphi_{Q_{i, k}}\right\rangle\right|\left(\left|\partial^{\gamma} \psi_{Q_{i, k}}(x)\right|+\left|\partial^{\gamma} \psi_{Q_{i, k}}(y)\right|\right) \\
\leq & C \sum_{i=\max (L+1, l)}^{\infty} \sum_{k \in \mathbb{Z}^{n}}\left(1+\left|2^{-i} k\right|\right)^{-\alpha-n-\epsilon} 2^{-i(\alpha+n / 2)} \\
& \times 2^{i n / 2+[\beta] i}\left[\left(1+2^{i}\left|x-2^{-i} k\right|\right)^{-\alpha-n-\epsilon}+\left(1+2^{i}\left|y-2^{-i} k\right|\right)^{-\alpha-n-\epsilon}\right] \\
\leq & C\left(\sum_{i=\max (L+1, l)}^{\infty} 2^{-i(\alpha-[\beta])}\right) \sup _{|z| \leq|x-y|} \frac{1}{(1+|x-z|)^{\alpha+n+\epsilon}} .
\end{aligned}
$$


Since $\alpha>\beta,|\gamma|=[\beta]$ and

$$
\begin{aligned}
\sum_{i=\max (L+1, l)}^{\infty} 2^{-i(\alpha-[\beta])} & \leq\left(\sum_{i=L+1}^{\infty} 2^{-2 i(\alpha-\beta)}\right)^{1 / 2}\left(\sum_{i=l}^{\infty} 2^{-2 i(\beta-[\beta])}\right)^{1 / 2} \\
& \leq C 2^{(-L-1)(\alpha-\beta)} 2^{-l(\beta-[\beta])} \\
& \leq C 2^{(-L-1)(\alpha-\beta)}|x-y|^{\beta-[\beta]}
\end{aligned}
$$

we assert that

$$
I I I^{\prime} \leq C 2^{(-L-1)(\alpha-\beta)}|x-y|^{\beta-[\beta]} \sup _{|z| \leq|x-y|} \frac{1}{(1+|x-z|)^{\beta+n+\epsilon}} .
$$

For $I V^{\prime}$ and $V^{\prime}$, we use the following condition satisfied by $\psi_{Q_{i, k}}$ that

$$
\begin{aligned}
& \left|\partial^{\gamma} \psi_{Q_{i, k}}(x)-\partial^{\gamma} \psi_{Q_{i, k}}(y)\right| \\
& \quad \leq 2^{i(n / 2+\beta)}|x-y|^{\beta-[\beta]} \sup _{\left|z-2^{-i} k\right| \leq|x-y|} \frac{1}{\left(1+2^{i}|x-z|\right)^{\beta+n+\epsilon}} \\
& \quad \leq C 2^{i(n / 2+\beta)}|x-y|^{\beta-[\beta]}\left(\frac{1}{1+2^{i}\left|x-2^{-i} k\right|}\right)^{\beta+n+\epsilon}
\end{aligned}
$$

for $\left|z-2^{-i} k\right| \leq|x-y| \leq 2^{-l+1} \leq 2^{-i}$. Recall that $I V^{\prime}$ vanishes unless $\max (L+1, l)=l$. Thus, when we estimate $I V^{\prime}$, we have $L+1 \leq i \leq$ $\max (L+1, l)-1=l-1$ and, hence, $2^{-l+1} \leq 2^{-i}$.

For $I V^{\prime}$, by a similar estimate as $I^{\prime}$, we have

$$
\begin{aligned}
I V^{\prime} & \leq C\left(\sum_{i=L+1}^{\infty} 2^{-i(\alpha-\beta)}\right)|x-y|^{\beta-[\beta]}\left(\frac{1}{1+|x|}\right)^{\alpha+n+\epsilon} \\
& \leq C 2^{(-L-1)(\alpha-\beta)}|x-y|^{\beta-[\beta]} \sup _{|z| \leq|x-y|} \frac{1}{(1+|x-z|)^{\beta+n+\epsilon}}
\end{aligned}
$$

as $\alpha>\beta$.

For $V^{\prime}$, using the idea for estimating $I I^{\prime}$, we obtain

$$
\begin{aligned}
V^{\prime} & \leq C\left(\sum_{i=-\infty}^{-L^{\prime}-1} 2^{i(\beta+\alpha-\beta-\epsilon)}\right)|x-y|^{\beta-[\beta]}\left(\frac{1}{1+|x|}\right)^{\beta+n+\epsilon} \\
& \leq C 2^{\left(-L^{\prime}-1\right)(\alpha-\epsilon)}|x-y|^{\beta-[\beta]} \sup _{|z| \leq|x-y|} \frac{1}{(1+|x-z|)^{\beta+n+\epsilon}},
\end{aligned}
$$


because $\alpha>\beta+\epsilon$.

We do not have $I I I^{\prime \prime}$ when $L=\min (l, L)$. Hence, we find that

$$
I I I^{\prime \prime}+I V^{\prime \prime}+V^{\prime \prime} \leq C 2^{-L^{\prime \prime}(\alpha-\beta)}|x-y|^{\beta-[\beta]} \sup _{|z| \leq|x-y|} \frac{1}{(1+|x-z|)^{\beta+n+\epsilon}} .
$$

Therefore, we assert that

$$
\begin{aligned}
& \left\|\sum_{i=-N^{\prime}}^{N} \sum_{|k| \leq \delta(i) 2^{N^{\prime \prime}}}\left\langle g, \varphi_{Q_{i, k}}\right\rangle \psi_{Q_{i, k}}-\sum_{i=-L^{\prime}}^{L} \sum_{|k| \leq \delta(i) 2^{L^{\prime \prime}}}\left\langle g, \varphi_{Q_{i, k}}\right\rangle \psi_{Q_{i, k}}\right\|_{\beta * *} \\
& \quad \leq C\left(2^{(-L-1)(\alpha-\beta)}+2^{\left(-L^{\prime}-1\right)(\alpha-\epsilon)}+2^{-L^{\prime \prime}(\alpha-\beta)}\right) .
\end{aligned}
$$

Since $\|\cdot\|_{\mathcal{C}_{\beta}}=\max \left(\|\cdot\|_{\beta *},\|\cdot\|_{\beta * *}\right)$, by (7.5) and (7.9), in general (without the assumptions $\|g\|_{\mathcal{C}_{\alpha}}=1, N>L, N^{\prime}>L^{\prime}$ and $N^{\prime \prime}>L^{\prime \prime}$ ), we establish the estimate,

$$
\begin{gathered}
\left\|\sum_{i=-N^{\prime}}^{N} \sum_{|k| \leq \delta(i) 2^{N^{\prime \prime}}}\left\langle g, \varphi_{Q_{i, k}}\right\rangle \psi_{Q_{i, k}}-\sum_{i=-L^{\prime}}^{L} \sum_{|k| \leq \delta(i) 2^{L^{\prime \prime}}}\left\langle g, \varphi_{Q_{i, k}}\right\rangle \psi_{Q_{i, k}}\right\|_{\mathcal{C}_{\beta}} \\
\leq C\|g\|_{\mathcal{C}_{\alpha}}\left(2^{(-\min (L, N)-1)(\alpha-\beta)}+2^{\left(-\min \left(L^{\prime}, N^{\prime}\right)-1\right)(\alpha-\beta-\epsilon)}\right. \\
\left.+2^{-\min \left(L^{\prime \prime}, N^{\prime \prime}\right)(\alpha-\beta)}\right) .
\end{gathered}
$$

Hence, $\sum_{i=-L^{\prime}}^{L} \sum_{|k| \leq \delta(i) 2^{L^{\prime \prime}}}\left\langle g, \varphi_{Q_{i, k}}\right\rangle \psi_{Q_{i, k}}(x)$ is a Cauchy sequence in $\mathcal{C}_{\beta}\left(\mathbb{R}^{n}\right)$. As $\mathcal{C}_{\beta}\left(\mathbb{R}^{n}\right) \subset L^{2}\left(\mathbb{R}^{n}\right)$, we represent $g$ by the frame expansion, $g=$ $\sum_{i \in \mathbb{Z}, k \in \mathbb{Z}^{n}}\left\langle g, \varphi_{Q_{i, k}}\right\rangle \psi_{Q_{i, k}}$, and the above identity holds in $L^{2}\left(\mathbb{R}^{n}\right)$. The limit in $\mathcal{C}_{\beta}\left(\mathbb{R}^{n}\right)$ equals to the limit in $L^{2}\left(\mathbb{R}^{n}\right)$. Therefore, $\lim _{L, L^{\prime}, L^{\prime \prime} \rightarrow \infty} \sum_{i=-L^{\prime}}^{L}$ $\sum_{|k| \leq \delta(i) 2^{L^{\prime \prime}}}\left\langle g, \varphi_{Q_{i, k}}\right\rangle \psi_{Q_{i, k}}=g$ in $\mathcal{C}_{\beta}\left(\mathbb{R}^{n}\right)$.

Let $N, N^{\prime}$ and $N^{\prime \prime}$ go to infinity in (7.10), we have

$$
\begin{aligned}
& \left\|g-\sum_{i=-L^{\prime}}^{L} \sum_{|k| \leq \delta(i) 2^{L^{\prime \prime}}}\left\langle g, \varphi_{Q_{i, k}}\right\rangle \psi_{Q_{i, k}}\right\|_{\mathcal{C}_{\beta}} \\
& \quad \leq C\|g\|_{\mathcal{C}_{\alpha}}\left(2^{(-L-1)(\alpha-\beta)}+2^{\left(-L^{\prime}-1\right)(\alpha-\beta-\epsilon)}+2^{-L^{\prime \prime}(\alpha-\beta)}\right) .
\end{aligned}
$$

We prove the first part of Theorem 4.1.

The second part of Theorem 4.1 is a straightforward consequence of the fact, $\bigcap_{\alpha>0} \mathcal{C}_{\alpha}\left(\mathbb{R}^{n}\right)=\mathcal{S}\left(\mathbb{R}^{n}\right)$, and the first part of this theorem. 


\section{§. Appendix}

\subsection{Truncation sets}

We consider the convergence of the following frame expansions for $f \in$ $\mathcal{S}^{\prime}\left(\mathbb{R}^{n}\right)$

$$
\sum_{-L^{\prime} \leq i \leq L} \sum_{|k| \leq 2^{i+L^{\prime \prime}}}\left\langle f, \psi_{Q_{i, k}}\right\rangle \varphi_{Q_{i, k}} \text { and } \sum_{-L^{\prime} \leq i \leq L} \sum_{|k| \leq 2^{L^{\prime \prime}}}\left\langle f, \psi_{Q_{i, k}}\right\rangle \varphi_{Q_{i, k}} .
$$

That is, the truncation sets are

$$
\tilde{T}\left(L, L^{\prime}, L^{\prime \prime}\right)=\left\{(i, k): i \in \mathbb{Z},-L^{\prime} \leq i \leq L ; k \in \mathbb{Z}^{n},\left|2^{-i} k\right| \leq 2^{L^{\prime \prime}}\right\},
$$

and

$$
\hat{T}\left(L, L^{\prime}, L^{\prime \prime}\right)=\left\{(i, k): i \in \mathbb{Z},-L^{\prime} \leq i \leq L ; k \in \mathbb{Z}^{n},|k| \leq 2^{L^{\prime \prime}}\right\},
$$

respectively.

No matter we take $T\left(L, L^{\prime}, L^{\prime \prime}\right), \tilde{T}\left(L, L^{\prime}, L^{\prime \prime}\right)$ or $\hat{T}\left(L, L^{\prime}, L^{\prime \prime}\right)$ as the truncation set, we obtain the same estimates for $I^{\prime}, I I^{\prime}, I I I^{\prime}, I V^{\prime}$ and $V^{\prime}$. The differences are found in $I^{\prime \prime}, I I^{\prime \prime}, I I I^{\prime \prime}, I V^{\prime \prime}$ and $V^{\prime \prime}$. For simplicity, we just discuss the estimate of $I I^{\prime \prime}$ with $\tilde{T}\left(L, L^{\prime}, L^{\prime \prime}\right)$ as the truncation set and the estimate of $I^{\prime \prime}$ with $\hat{T}\left(L, L^{\prime}, L^{\prime \prime}\right)$ as the truncation set.

If we use $\tilde{T}\left(L, L^{\prime}, L^{\prime \prime}\right)$ as the truncation set, then, for any fixed $\tilde{\alpha}$ satisfying $\alpha>\tilde{\alpha}+\epsilon>\beta+\epsilon$, we find that for any $\gamma \in \mathbb{N}^{n}$ and $0 \leq|\gamma| \leq[\beta]$,

$$
\begin{aligned}
I I^{\prime \prime}= & \sum_{i=-L^{\prime}}^{-1} \sum_{|k|>2^{i+L^{\prime \prime}}}\left|\left\langle g, \varphi_{Q_{i, k}}\right\rangle\right|\left|\partial^{\gamma} \psi_{Q_{i, k}}(x)\right| \\
\leq C & \sum_{i=-L^{\prime}}^{-1} \sum_{|k|>2^{i+L^{\prime \prime}}}\left(\frac{1}{1+|k|}\right)^{\tilde{\alpha}+n+\epsilon} 2^{i(\alpha+n / 2)} 2^{i n / 2+|\gamma| i} \\
& \times\left(\frac{1}{1+2^{i}\left|x-2^{-i} k\right|}\right)^{\alpha+n+\epsilon} .
\end{aligned}
$$

By using the fact that $|k|>2^{i+L^{\prime \prime}}$ and $\alpha>\tilde{\alpha}>\beta$, we have,

$$
\begin{aligned}
I I^{\prime \prime} \leq C \sum_{i=-L^{\prime}}^{-1} \sum_{|k|>2^{i+L^{\prime \prime}}}\left(\frac{1}{1+2^{i+L^{\prime \prime}}}\right)^{\tilde{\alpha}-\beta}\left(\frac{1}{1+|k|}\right)^{\beta+n+\epsilon} 2^{i(n+\alpha+|\gamma|)} \\
\quad \times\left(\frac{1}{1+2^{i}\left|x-2^{-i} k\right|}\right)^{\beta+n+\epsilon}
\end{aligned}
$$


As $i<0$, we obtain

$$
\begin{aligned}
I I^{\prime \prime} & \leq C \sum_{i=-L^{\prime}}^{-1} 2^{i(n+|\gamma|+\alpha)} 2^{\left(i+L^{\prime \prime}\right)(\beta-\tilde{\alpha})}\left(\frac{1}{1+2^{i}|x|}\right)^{\beta+n+\epsilon} \\
& \leq C \sum_{i=-L^{\prime}}^{-1} 2^{i(n+|\gamma|+\alpha)} 2^{\left(i+L^{\prime \prime}\right)(\beta-\tilde{\alpha})}\left(\frac{2^{-i}}{2^{-i}+|x|}\right)^{\beta+n+\epsilon} \\
& \leq C \sum_{i=-L^{\prime}}^{-1} 2^{i(n+|\gamma|+\alpha-\beta-n-\epsilon)} 2^{\left(i+L^{\prime \prime}\right)(\beta-\tilde{\alpha})}\left(\frac{1}{1+|x|}\right)^{\beta+n+\epsilon},
\end{aligned}
$$

and finally, we assert that

$$
\begin{aligned}
I I^{\prime \prime} & \leq C \sum_{i=-L^{\prime}}^{-1} 2^{i(|\gamma|+\alpha-\tilde{\alpha}-\epsilon)} 2^{L^{\prime \prime}(\beta-\tilde{\alpha})}\left(\frac{1}{1+|x|}\right)^{\beta+n+\epsilon} \\
& \leq C 2^{-L^{\prime \prime}(\tilde{\alpha}-\beta)}\left(\frac{1}{1+|x|}\right)^{\beta+n+\epsilon}
\end{aligned}
$$

because $0 \leq|\gamma| \leq[\beta]$ and $\alpha>\tilde{\alpha}+\epsilon$.

Thus, for any $\alpha>\tilde{\alpha}+\epsilon>\beta+\epsilon$, instead of (7.11), we have a constant $C>0$ independent of $L, L^{\prime}$ and $L^{\prime \prime}$ such that, for any $g \in \mathcal{C}_{\alpha}\left(\mathbb{R}^{n}\right)$ satisfying $(4.7)$,

$$
\begin{aligned}
& \left\|g-\sum_{i=-L^{\prime}}^{L} \sum_{|k| \leq 2^{i+L^{\prime \prime}}}\left\langle g, \varphi_{Q_{i, k}}\right\rangle \psi_{Q_{i, k}}\right\|_{\mathcal{C}_{\beta}} \\
& \quad \leq C\|g\|_{\mathcal{C}_{\alpha}}\left(2^{(-L-1)(\alpha-\beta)}+2^{\left(-L^{\prime}-1\right)(\alpha-\beta-\epsilon)}+2^{-L^{\prime \prime}(\tilde{\alpha}-\beta)}\right) .
\end{aligned}
$$

Now, we turn to the convergence of frame expansion with $\hat{T}\left(L, L^{\prime}, L^{\prime \prime}\right)$ as the truncation set. For any fixed $\tilde{\alpha}$ satisfying $\alpha>\tilde{\alpha}>\beta$ and any $0 \leq|\gamma| \leq[\beta]$, we estimate $I^{\prime \prime}$ by

$$
\begin{aligned}
I^{\prime \prime} \leq C \sum_{i=0}^{L} \sum_{|k|>2^{L^{\prime \prime}}}\left(1+\left|2^{-i} k\right|\right)^{-\alpha-n-\epsilon} 2^{-i \alpha-i n / 2} 2^{i n / 2+|\gamma| i} \\
\quad \times\left(1+2^{i}\left|x-2^{-i} k\right|\right)^{-\alpha-n-\epsilon} \\
\leq C \sum_{i=0}^{L} 2^{-i(\alpha-|\gamma|)} 2^{i(\alpha-\tilde{\alpha})} 2^{-L^{\prime \prime}(\alpha-\tilde{\alpha})} \sum_{k \in \mathbb{Z}^{n}}\left(1+\left|2^{-i} k\right|\right)^{-\tilde{\alpha}-n-\epsilon} \\
\times\left(1+2^{i}\left|x-2^{-i} k\right|\right)^{-\alpha-n-\epsilon},
\end{aligned}
$$


since $|k|>2^{L^{\prime \prime}}>1$.

By the second part of Lemma 7.2, we find that

$$
\begin{aligned}
I^{\prime \prime} & \leq C\left(\sum_{i=0}^{L} 2^{-i(\tilde{\alpha}-|\gamma|)}\right) 2^{-L^{\prime \prime}(\alpha-\tilde{\alpha})}(1+|x|)^{-\beta-n-\epsilon} \\
& \leq C 2^{-L^{\prime \prime}(\alpha-\tilde{\alpha})}(1+|x|)^{-\beta-n-\epsilon}
\end{aligned}
$$

because $\alpha>\tilde{\alpha}>\beta$.

So, if we consider $\hat{T}\left(L, L^{\prime}, L^{\prime \prime}\right)$ as the truncation set, then, for any $\alpha>$ $\tilde{\alpha}>\beta$, we obtain a constant $C>0$ independent of $L, L^{\prime}$ and $L^{\prime \prime}$ such that, for any $g \in \mathcal{C}_{\alpha}\left(\mathbb{R}^{n}\right)$ satisfying (4.7),

$$
\begin{aligned}
& \left\|g-\sum_{i=-L^{\prime}}^{L} \sum_{|k| \leq 2^{L^{\prime \prime}}}\left\langle g, \varphi_{Q_{i, k}}\right\rangle \psi_{Q_{i, k}}\right\|_{\mathcal{C}_{\beta}} \\
& \leq C\|g\|_{\mathcal{C}_{\alpha}}\left(2^{(-L-1)(\alpha-\beta)}+2^{\left(-L^{\prime}-1\right)(\alpha-\beta-\epsilon)}+2^{-L^{\prime \prime}(\alpha-\tilde{\alpha})}\right) .
\end{aligned}
$$

Comparing (8.1) and (8.2) to (7.11), the truncated frame expansions using $\tilde{T}\left(L, L^{\prime}, L^{\prime \prime}\right)$ and $\hat{T}\left(L, L^{\prime}, L^{\prime \prime}\right)$ as the truncation sets converge slower than the frame expansion truncated by $T\left(L, L^{\prime}, L^{\prime \prime}\right)$. This is expected since, for any fixed $L, L^{\prime}, L^{\prime \prime}>0, \tilde{T}\left(L, L^{\prime}, L^{\prime \prime}\right)$ and $\hat{T}\left(L, L^{\prime}, L^{\prime \prime}\right)$ are proper subsets of $T\left(L, L^{\prime}, L^{\prime \prime}\right)$.

In addition, the upper bound of $\alpha-\tilde{\alpha}$ in (8.2) is $\alpha-\beta$, but the upper bound of $\tilde{\alpha}-\beta$ in (8.1) equals to $\alpha-\epsilon-\beta$. This reflects that the truncated frame expansion, (8.2), converges more rapidly than (8.1).

With some easy modifications on Theorem 2.1 and Theorem 4.1, we have the following extension of Theorem 2.1.

Theorem 8.1. Let $\left\{\varphi_{Q}\right\}_{Q \in \mathcal{Q}} \in \bigcap_{\alpha>0} \mathcal{M}_{\alpha}\left(\mathbb{R}^{n}\right)$ be a frame on $L^{2}\left(\mathbb{R}^{n}\right)$ and $\left\{\psi_{Q}\right\}_{Q \in \mathcal{Q}} \in \bigcap_{\alpha>0} \mathcal{M}_{\alpha}\left(\mathbb{R}^{n}\right)$ be its dual frame, then, for any $f \in \mathcal{S}^{\prime}\left(\mathbb{R}^{n}\right)$ of order $\omega$, there exist two sequences of polynomials, $\tilde{P}_{L, L^{\prime}, L^{\prime \prime}}$ and $\hat{P}_{L, L^{\prime}, L^{\prime \prime}}$, of degrees less than or equal to $[\omega]$ such that

$$
\lim _{L, L^{\prime}, L^{\prime \prime} \rightarrow \infty}\left\{\sum_{-L^{\prime} \leq i \leq L} \sum_{|k| \leq 2^{i+L^{\prime \prime}}}\left\langle f, \psi_{Q_{i, k}}\right\rangle \varphi_{Q_{i, k}}-\tilde{P}_{L, L^{\prime}, L^{\prime \prime}}\right\}=f,
$$

and

$$
\lim _{L, L^{\prime}, L^{\prime \prime} \rightarrow \infty}\left\{\sum_{-L^{\prime} \leq i \leq L} \sum_{|k| \leq 2^{L^{\prime \prime}}}\left\langle f, \psi_{Q_{i, k}}\right\rangle \varphi_{Q_{i, k}}-\hat{P}_{L, L^{\prime}, L^{\prime \prime}}\right\}=f
$$


in $\mathcal{S}^{\prime}\left(\mathbb{R}^{n}\right)$.

The corresponding generalizations for the other theorems are left to the reader.

\subsection{Floating polynomials}

In this section, we first show that the convergence in (2.4) is independent of the family of functions, $\left\{\theta_{\lambda}\right\}_{\lambda} \subset \mathcal{S}\left(\mathbb{R}^{n}\right)$, that satisfies (2.5).

Proposition 8.2. Suppose that $\left\{\theta_{\lambda}^{1}\right\}$ and $\left\{\theta_{\lambda}^{2}\right\}$ are two families of Schwartz functions satisfying (2.5) and $f \in \mathcal{S}^{\prime}\left(\mathbb{R}^{n}\right)$ is of order $\omega$. Let

$$
\begin{array}{r}
P_{L, L^{\prime}, L^{\prime \prime}}^{\theta^{j}}(x)=-\sum_{0 \leq|\lambda| \leq[\omega]}\left(\left\langle f, \theta_{\lambda}^{j}\right\rangle-\sum_{-L^{\prime} \leq i \leq L} \sum_{|k| \leq \delta(i) L^{L^{\prime \prime}}}\left\langle\varphi_{Q_{i, k}}, \theta_{\lambda}^{j}\right\rangle\left\langle f, \psi_{Q_{i, k}}\right\rangle\right) x^{\lambda}, \\
j=1,2,
\end{array}
$$

be the floating polynomials corresponding to $\left\{\theta_{\lambda}^{j}\right\}, j=1,2$, respectively. Then, on any compact set of $\mathbb{R}^{n}$,

$$
\lim _{L, L^{\prime}, L^{\prime \prime} \rightarrow \infty}\left(P_{L, L^{\prime}, L^{\prime \prime}}^{\theta^{1}}-P_{L, L^{\prime}, L^{\prime \prime}}^{\theta^{2}}\right)=0
$$

uniformly and, hence, $P_{L, L^{\prime}, L^{\prime \prime}}^{\theta^{1}}-P_{L, L^{\prime}, L^{\prime \prime}}^{\theta^{2}}$ converges to zero in $\mathcal{S}^{\prime}\left(\mathbb{R}^{n}\right)$ as $L$, $L^{\prime}$ and $L^{\prime \prime}$ go to infinity.

Proof. We only need to show that the coefficient of $x^{\lambda}$ for $P_{L, L^{\prime}, L^{\prime \prime}}^{\theta^{1}}(x)$ converges to the coefficient of $x^{\lambda}$ for $P_{L, L^{\prime}, L^{\prime \prime}}^{\theta^{2}}(x)$. That is, for any $f \in \mathcal{S}^{\prime}\left(\mathbb{R}^{n}\right)$ of order $\omega$,

$$
\begin{aligned}
& \lim _{L, L^{\prime}, L^{\prime \prime} \rightarrow \infty}\left(\left\langle f, \theta_{\lambda}^{1}\right\rangle-\sum_{-L^{\prime} \leq i \leq L} \sum_{|k| \leq \delta(i) 2^{L^{\prime \prime}}}\left\langle\varphi_{Q_{i, k}}, \theta_{\lambda}^{1}\right\rangle\left\langle f, \psi_{Q_{i, k}}\right\rangle\right) \\
& =\lim _{L, L^{\prime}, L^{\prime \prime} \rightarrow \infty}\left(\left\langle f, \theta_{\lambda}^{2}\right\rangle-\sum_{-L^{\prime} \leq i \leq L} \sum_{|k| \leq \delta(i) 2^{L^{\prime \prime}}}\left\langle\varphi_{Q_{i, k}}, \theta_{\lambda}^{2}\right\rangle\left\langle f, \psi_{Q_{i, k}}\right\rangle\right) .
\end{aligned}
$$

According to (2.5) with $N=[\omega]$, for any $\lambda \in \mathbb{N}^{n}$ and $0 \leq|\lambda| \leq[\omega]$, the function, $\Theta_{\lambda}(x)=\theta_{\lambda}^{1}(x)-\theta_{\lambda}^{2}(x)$, belongs to $\mathcal{S}\left(\mathbb{R}^{n}\right)$ and satisfies

$$
\int_{\mathbb{R}^{n}} x^{\gamma} \Theta_{\lambda}(x) d x=0 \quad \text { for } \gamma \in \mathbb{N}^{n} \text { and } 0 \leq|\gamma| \leq[\omega] .
$$


Consider the family, $\mathcal{C}_{\alpha}\left(\mathbb{R}^{n}\right)$, with $\epsilon=([\omega]+1-\omega) / 2$. By Theorem 4.1, we find that

$$
\lim _{L, L^{\prime}, L^{\prime \prime} \rightarrow \infty} \sum_{-L^{\prime} \leq i \leq L} \sum_{|k| \leq \delta(i) 2^{L^{\prime \prime}}}\left\langle\Theta_{\lambda}, \varphi_{Q_{i, k}}\right\rangle \psi_{Q_{i, k}}=\Theta_{\lambda}
$$

in $\mathcal{C}_{\eta}\left(\mathbb{R}^{n}\right)$, for any $\eta>\omega$ satisfying $[\eta]=[\omega]$ and $[\omega]+1>\eta+\epsilon$. Applying $f$ on both sides of (8.6), we obtain

$$
\begin{aligned}
& \lim _{L, L^{\prime}, L^{\prime \prime} \rightarrow \infty}\left(\sum_{-L^{\prime} \leq i \leq L} \sum_{|k| \leq \delta(i) 2^{L^{\prime \prime}}}\left\langle\varphi_{Q_{i, k}}, \theta_{\lambda}^{1}\right\rangle\left\langle f, \psi_{Q_{i, k}}\right\rangle\right. \\
&\left.-\sum_{-L^{\prime} \leq i \leq L} \sum_{|k| \leq \delta(i) 2^{L^{\prime \prime}}}\left\langle\varphi_{Q_{i, k}}, \theta_{\lambda}^{2}\right\rangle\left\langle f, \psi_{Q_{i, k}}\right\rangle\right) \\
&= \lim _{L, L^{\prime}, L^{\prime \prime} \rightarrow \infty} \sum_{-L^{\prime} \leq i \leq L} \sum_{|k| \leq \delta(i) 2^{L^{\prime \prime}}}\left\langle\varphi_{Q_{i, k}}, \Theta_{\lambda}\right\rangle\left\langle f, \psi_{Q_{i, k}}\right\rangle \\
&=\left\langle f, \Theta_{\lambda}\right\rangle=\left\langle f, \theta_{\lambda}^{1}\right\rangle-\left\langle f, \theta_{\lambda}^{2}\right\rangle,
\end{aligned}
$$

which is our desired result.

The following proposition shows that Theorem 2.1 is consistent with the well-known convergence of frame expansion for functions in $L^{2}\left(\mathbb{R}^{n}\right)$.

Proposition 8.3. If $f \in L^{2}\left(\mathbb{R}^{n}\right)$, then the corresponding sequence of floating polynomials,

$$
P_{L, L^{\prime}, L^{\prime \prime}}(x)=-\sum_{0 \leq \lambda \leq N}\left(\left\langle f, \theta_{\lambda}\right\rangle-\sum_{-L^{\prime} \leq i \leq L} \sum_{|k| \leq \delta(i) 2^{L^{\prime \prime}}}\left\langle\varphi_{Q_{i, k}}, \theta_{\lambda}\right\rangle\left\langle f, \psi_{Q_{i, k}}\right\rangle\right) x^{\lambda},
$$

converges to zero in $\mathcal{S}^{\prime}\left(\mathbb{R}^{n}\right)$ as $L, L^{\prime}$ and $L^{\prime \prime}$ go to infinity.

Proof. It is sufficient to show that

$$
\lim _{L, L^{\prime}, L^{\prime \prime} \rightarrow \infty}\left(\left\langle f, \theta_{\lambda}\right\rangle-\sum_{-L^{\prime} \leq i \leq L} \sum_{|k| \leq \delta(i) 2^{L^{\prime \prime}}}\left\langle\varphi_{Q_{i, k}}, \theta_{\lambda}\right\rangle\left\langle f, \psi_{Q_{i, k}}\right\rangle\right)=0 .
$$

By the Cauchy-Schwartz inequality, we find that

$$
\begin{aligned}
& \left|\left\langle f, \theta_{\lambda}\right\rangle-\sum_{-L^{\prime} \leq i \leq L} \sum_{|k| \leq \delta(i) 2^{L^{\prime \prime}}}\left\langle\varphi_{Q_{i, k}}, \theta_{\lambda}\right\rangle\left\langle f, \psi_{Q_{i, k}}\right\rangle\right| \\
& \quad \leq\left\|f-\sum_{-L^{\prime} \leq i \leq L} \sum_{|k| \leq \delta(i) 2^{L^{\prime \prime}}}\left\langle f, \psi_{Q_{i, k}}\right\rangle \varphi_{Q_{i, k}}\right\|_{L^{2}\left(\mathbb{R}^{n}\right)}\left\|\theta_{\lambda}\right\|_{L^{2}\left(\mathbb{R}^{n}\right)} .
\end{aligned}
$$


Since $\left\{\varphi_{Q_{i, k}}\right\}_{i \in \mathbb{Z}, k \in \mathbb{Z}^{n}}$ is an frame on $L^{2}\left(\mathbb{R}^{n}\right)$ and $\left\{\psi_{Q_{i, k}}\right\}_{i \in \mathbb{Z}, k \in \mathbb{Z}^{n}}$ is its dual frame, we have

$$
\left\|f-\sum_{-L^{\prime} \leq i \leq L} \sum_{|k| \leq \delta(i) 2^{L^{\prime \prime}}}\left\langle f, \psi_{Q_{i, k}}\right\rangle \varphi_{Q_{i, k}}\right\|_{L^{2}\left(\mathbb{R}^{n}\right)} \rightarrow 0
$$

as $L, L^{\prime}$ and $L^{\prime \prime}$ go to infinity, and, hence, we obtain our desired result.

\section{REFERENCES}

[1] R. Coifman and Y. Meyer, Wavelets: Calderón-Zygmund and Multilinear Operators, Cambridge studies in adv. math., \#48, Cambridge Univ. Press, 1997.

[2] J. Conway, A Course in Functional Analysis, Graduate texts in mathematics, \#96, Springer-Verlag, 1990.

[3] I. Daubechies, Ten Lectures on Wavelets, CBMS-NSF regional conference series in applied mathematics \#61, Society for Industrial and Applied Mathematics, 1992.

[4] G. Fix and G. Strang, A Fourier analysis of the finite element variational method, Construct. Aspects of Funct. Anal. (1971), 796-830.

[5] M. Frazier and B. Jawerth, Decomposition of Besov spaces, Indiana Univ. Math., 34 (1985), 777-799.

[6] M. Frazier and B. Jawerth, A Discrete Transform and Decomposition of Distribution Spaces, J. Funct. Anal., 93 (1990), 34-170.

[7] M. Frazier, B. Jawerth and G. Weiss, Littlewood-Paley Theory and the Study of Function Spaces, CBMS Regional Conference Ser., \#79, American Math. Society, 1991.

[8] E. Hernández and G. Weiss, A first Course on Wavelets, CRC Press, 1996.

[9] K.-P. Ho, Frame associated with Expansive Matrix Dilation, Collect. Math., 54 (2003), 217-254.

[10] K.-P. Ho, Remarks on Littlewood-Paley analysis, Canad. J. Math., to appear.

[11] S. Jaffard and Y. Meyer, Wavelet Methods for Pointwise Regularity and Local Oscillations of Functions, Mem. Amer. Math. Soc., \#123, 1996.

[12] S. Kelly, M. Kon and L. Raphael, Pointwise convergence of wavelet expansions, Bull. Amer. Math. Soc. (N.S.), 30 (1994), no. 1, 87-94.

[13] S. Kelly, M. Kon and L. Raphael, Local convergence for wavelet expansions, J. Funct. Anal., 126 (1994), no. 1, 102-138.

[14] S. Mallat, A Wavelet Tour of Signal Processing, Academic Press, 1999.

[15] Y. Meyer, Wavelets and Operators, Cambridge studies in adv. math., \#37, Cambridge Univ. Press, 1992.

[16] Y. Meyer, Wavelets, Vibrations and Scalings, CRM Monograph Series, \#9, AMS, 1998.

[17] Y. Meyer, Oscillating Patterns in Image Processing and Nonlinear Evolution Equations, University Lecture Series, \#22, AMS, 2001. 
[18] J. Peetre, New thoughts on Besov spaces, Duke University Mathematics Series \#1, Mathematics Depatrment, Duke University, 1976.

[19] I. Singer, Bases in Banach spaces I, Springer-Verlag, 1970.

[20] F. Treves, Topological Vector Spaces, Distributions and Kernels, Pure and Applied Maths., \#25, Academic Press, 1967.

[21] D. Walnut, An Introduction to Wavelet Analysis, Birkhauser, 2002.

[22] G. Walter, Pointwise convergence of wavelet expansions, J. Approx. Theory, 80 (1995), no. 1, 108-118.

[23] G. Walter, Wavelets and generalized functions. Wavelets: A Tutorial in Theory and Application, Wavelet Anal. Appl., \#2, Academic Press, 1992, pp. 51-70.

[24] P. Wojtaszczyk, A Mathematical Introduction to Wavelets, Cambridge University Press, 1997.

[25] R. Young, An introduction to nonharmonic Fourier series, Academic Press, 2001.

[26] A. Zayed, Pointwise convergence of a class of non-orthogonal wavelet expansions, Proc. Amer. Math. Soc., 128 (2000), no. 12, 3629-3637.

Department of Mathematics

Hong Kong University of Science and Technology

Clear Water Bay

Hong Kong

China

makho@ust.hk 